\title{
A deep learning framework for elucidating whole-genome chemical interaction space
}

\section{Tian Cai ${ }^{1}$, Hansaim $\mathrm{Lim}^{2}$, Kyra Alyssa Abbu ${ }^{3}$, Yue Qiu ${ }^{4}$, Ruth Nussinov ${ }^{5,6}$, and Lei Xie ${ }^{1,2,3,4,7, *}$}

\footnotetext{
${ }^{1}$ Ph.D. Program in Computer Science, The Graduate Center, The City University of New York, New York, 10016, USA

${ }^{2}$ Ph.D. Program in Biochemistry, The Graduate Center, The City University of New York, New York, 10016, USA

${ }^{3}$ Department of Computer Science, Hunter College, The City University of New York, New York, 10065, USA

${ }^{4}$ Ph.D. Program in Biology, The Graduate Center, The City University of New York, New York, 10016, USA

${ }^{5}$ Computational Structural Biology Section, Basic Science Program, Frederick National Laboratory for Cancer Research, Frederick, MD 21702, USA

${ }^{6}$ Department of Human Molecular Genetics and Biochemistry, Sackler School of Medicine, Tel Aviv University, Tel
Aviv, Israel

${ }^{7}$ Helen and Robert Appel Alzheimer's Disease Research Institute, Feil Family Brain 83 Mind Research Institute, Weill Cornell Medicine, Cornell University, New York, 10021, USA

* Corresponding Author: Lei Xie, Address: 1008N 695 Park Avenue, New York City, NY 10065, USA, Phone: 212-396-6550,Email: lei.xie@hunter.cuny.edu
}

October 9, 2020

Keywords - machine learning, self-supervised learning, protein sequence representation, chemicalprotein interaction, G-Protein Coupled Receptor, drug discovery 


\begin{abstract}
Molecular interaction is the foundation of biological process. Elucidation of genome-wide binding partners of a biomolecule will address many questions in biomedicine. However, ligands of a vast number of proteins remain elusive. Existing methods mostly fail when the protein of interest is dissimilar from those with known functions or structures. We develop a new deep learning framework DISAE that incorporates biological knowledge into self-supervised learning techniques for predicting ligands of novel unannotated proteins on a genome-scale. In the rigorous benchmark studies, DISAE outperforms state-of-the-art methods by a significant margin. The interpretability analysis of DISAE suggests that it learns biologically meaningful information. We further use DISAE to assign ligands to human orphan G-Protein Coupled Receptors (GPCRs) and to cluster the human GPCRome by integrating their phylogenetic and ligand relationships. The promising results of DISAE open an avenue for exploring the chemical landscape of entire sequenced genomes.
\end{abstract}




\section{Introduction}

Small molecules like metabolites and drugs play an essential role in modulating physiological and pathological processes. The chemical modulation of biological system results from its interaction with biomolecules, largely proteins. Thus, the genome-wide identification of chemical-protein interactions (CPI) will not only address many fundamental questions in biology (e.g. microbiome-host interaction mediated by the metabolite) but also provide new opportunities in drug discovery and precision medicine [1. In spite of tremendous advances in genomics, the function of a vast number of proteins, particularly, their ligands are to a great extent unknown. Among approximately 3,000 druggable genes that encode human proteins, only $5 \%$ to $10 \%$ of them have been targeted by an FDA-approved drug 2 . The proteins that miss ligand information are called orphan proteins in biology or considered as unlabeled data in terms of machine learning. It is a great challenge to assign ligands to the orphan proteins especially when they are dissimilar from proteins with known structures or functions. Many experimental approaches have been developed to deorphanize the orphan proteins such as G-protein coupled receptors (GPCRs) 3]. However, they are costly and time-consuming. A great deal of efforts have been devoted to develop computational approaches, which may provide an efficient solution to generate testable hypotheses for elucidating the ligand of orphan proteins.

With the increasing availability of solved crystal structures of proteins, homology modeling and proteinligand docking are major methods for the effort in deorphanization 4. However, the quality of homology models significantly deteriorates when the sequence identity between query and template is low. When a homology model with an acceptable quality is unavailable, structure-based methods, either physics-based or machine learning-based [5] could be unfruitful. Moreover, protein-ligand docking suffers from a high rate of false positives because it is incapable of accurately modeling conformational dynamics, solvation effect, crystalized water molecules, and other physical phenomena. Considering that around half of Pfam families do not have any structure information [6], it is necessary to develop new sequence-based machine learning approaches to the deorphanization, especially for the proteins that are not close homologs of or even evolutionarily unrelated to the ones with solved crystal structures or known ligands.

Many machine learning methods have been developed to predict CPIs from protein sequences. Early works relied on feature engineering to create a representation of protein and ligand then use classifiers such as support vector machine [7 and matrix factorization 8 9] to make final prediction. End-to-end deep learning approaches have recently gained a momentum [10 11. Various neural network architectures, including Convolutional Neural Network (CNN) 12] 13, seq2seq [14] 15], and Transformer [16, have been applied to represent protein sequences. These works mainly focused on filling in missing CPIs for the existing drug targets. When the sequence of orphan protein is significantly different from those used in the database or training data, the performance of many existing machine learning methods deteriorates significantly [8].

To extend the scope of state-of-the-arts for the prediction of chemical binding to orphan proteins in entire sequenced genomes, we propose a new deep learning framework for improving protein representation learning so that the relationships between remote proteins or even evolutionary unrelated proteins can be detected. Inspired by the success in self-supervising learning of unlabeled data in Natural Language Processing (NLP) [17, and its application to biological sequences [18] [19] 20, we proposed a new protein representation method, DIstilled Sequence Alignment Embedding (DISAE), for the purpose of deorphanization of remote orphan proteins. Although the number of labeled proteins that have known ligands is limited, DISAE can utilize all available unlabeled protein sequences without the knowledge of their functions and structures. By incorporating biological knowledge into the sequence representation, DISAE can learn functionally important information about protein families that span a wide range of protein space. Furthermore, we devise a module-based pre-training-fine-tuning strategy using ALBERT [17]. To our knowledge, it is the first time to apply pre-trained-fine-tuned protein sequence model for addressing the challenge of deorphanization of novel proteins on a genome-scale.

In the benchmark study, DISAE significantly outperforms other state-of-the-art methods for the prediction of chemical binding to dissimilar orphan proteins with a large margin. Furthermore, The interpretability analysis of DISAE suggests that it learns biologically meaningful information. We apply DISAE to the deorphanization of G-protein coupled receptors (GPCRs). GPCRs play a pivotal role in numerous physiological and pathological processes. Due to their associations with many human diseases and high druggabilities, GPCRs are the most studied drug targets 21. Around one-third of FDA-approved drugs target GPCRs 21]. In spite of intensive studies in GPCRs, the endogenous and surrogate ligands of a large number of GPCRs remain unknown [3. Using DISAE, we can confidently assign 649 orphan GPCRs in Pfam with at least one ligand. 106 of the orphan GPCRs find at least one approved GPCR-targeted drugs as ligand with estimated false positive rate lower than 0.05. These predictions merit further experimental validations. In addition, we cluster the human GPCRome by integrating their sequence and ligand relationships. The promising results of DISAE open the revenue for exploring the chemical landscape of all sequenced genomes. The code of DISAE is available from github ${ }^{1}$

${ }^{1}$ https://github.com/XieResearchGroup/DISAE 


\section{Results and Discussion}

\subsection{Overview of methodology}

As illustrated in Figure 1 $\mathrm{A}$, the proposed method is designed to predict chemical binding to remote orphan proteins that do not have detectable relationships to annotated (i.e. labeled) proteins with known structures or functions. It is different from most of current works that focus on the assignment of functions to proteins that are homologous to the annotated proteins. Our method mainly consist of two stages (Figure 1B, C). The first stage is for unsupervised learning of protein representations using only sequence data from all non-redundant sequences in Pfam-A families 22] but without the need of any annotated structural or functional information. We develop a new algorithm, DIstilled Suquence Alignment Enmbedding (DISAE), for the self-supervised leaning (a special form of unspervised learing) of the protein representation. Different from existing sequence pre-training strategy that uses original protein sequences as input [18] 19] 20], DISAE distills the original sequence into an ordered list of triplets by excluding evolutionarily unimportant positions from a multiple sequence alignment (Figure 1B). The purpose of sequence distillation is two-folds: improving efficiency for the sequence pre-training in which long sequences cannot be handled, and reducing the noisy in the input sequence. Then long-range residue-residue interactions are learned via the Transformer module in ALBERT. A self-supervised masked language modeling (MLM) approach is used at this stage. In the MLM, $15 \%$ triplets are randomly masked and assumed that they are unknown. Then the remaining triplets are used to predict what the masked triplets are. In the second stage, a supervised learning model is trained to predict if a pair of chemical and protein interacts using the known CPIs that are collected from chemical genomics databases 23, 24, 25] 26. The input of the supervised learning includes both the representation of chemical structures from neural fingerprint [27] and the pre-trained protein sequence embedding from the first stage. We develop a module-based fine-tuning strategy to balance the information learned from the unsupervised and the supervised stages, and apply an attention pooling 28 to model the interaction between chemical substructures and protein residues. More details in the sequence representation learning, the architecture of neural network, benchmark data sets, training and evaluation procedure, and data analysis can be found in Method section.

\subsection{Pre-trained DISAE triplet vector is biochemically meaningful}

When pre-training ALBERT model with masked triplets of proteins, the masked word prediction accuracy reached 0.982 and 0.984 at the 80,000 th and the 100,000 th training step, respectively. For comparison, the pre-training accuracy when using BERT [29] was 0.955 at 200,000th training step. It may be because a larger batch size can be used in ALBERT than in BERT. The DIstilled Sequence Alignment Embedding (DISAE) from ALBERT pre-training is subsequently used for the CPI prediction. To evaluate whether the pre-trained DISAE vector is biochemically meaningful, we extracted the pre-trained DISAE vector for all possible triplets. We then used t-SNE to project the triplet vector in a $2 \mathrm{D}$ space. As shown in Supplementary Figure 1, the triplet vectors formed distinct clusters by the properties of amino acid side chains in the third amino acid of triplets, especially at level 3 and 4 . Triplets containing any ambiguous or uncommon amino acids, such as amino acid U for selenocysteine or X for any unresolved amino acids, formed a large cluster that did not form a smaller group (large group of black dots in each scatter plot), suggesting that the information regarding such rare amino acids are scarce in the pre-training dataset. When there is no ambiguity in triplets, they form clearly separated clusters, meaning that the pre-training process indeed allows the model to extract biochemically meaningful feature vectors from the proteins as sentences. The same clustering trends were also observed when triplets are grouped by individual amino acids, rather than their physicochemical properties of side chains (Supplementary Figure 2).

\subsection{DISAE significantly outperforms state-of-the-art models for predicting ligands of remote orphan proteins}

To evaluate the performance of DISAE for the prediction of ligand of remote orphan proteins, we perform experiments in a dissimilar protein benchmark. In this benchmark, the proteins in the testing data (i.e. query proteins) are significantly dissimilar from those in the training data (i.e. template proteins). As shown in Figure 2, the sequence identity of protein pairs between the query and the template are all less than $40 \%$. The pairs with the alignment length larger than 150 (around 50\% coverage of the protein) and the sequence identity larger than $30 \%$ only constitute less than $10 \%$ of total pairs. Thus, homology-based methods cannot be applied to majority of cases in the benchmark. We first evaluate the performance of two baseline models LSTM and Transformer in the dissimilar protein benchmark. In this conventional setting, the ALBERT model in $1 \mathrm{C}$ is replaced by LSTM or Transformer. In addition, there is no pre-training stage. Only labeled data are directly applied to supervised learning. As shown in Figure $3 \mathrm{~A}$ and $3 \mathrm{~B}$, the performance of conventional LSTM model is close to random. Its ROC-AUC and PR-AUC is 0.476 and 0.261, respectively. The Transformer-based architecture has achieved the state-of-the-art performance in the recently published work 16. Indeed, it outperforms LSTM model with a ROC-AUC of 0.648 and a PR-AUC of 0.380 , respectively. Interestingly, the ALBERT pre-trained model significantly improves the performance of state-of-the-arts. The ALBERT pre-trained model with the optimal configuration (transformer-frozen) 
has a ROC-AUC of 0.725 and a PR-AUC of 0.589 , respectively. Furthermore, as shown in the insert of Figure 3A, ALBERT pre-trained transformer-frozen model not only has the highest ROC-AUC and PRAUC but also far exceeds the baseline models at the range of low false positive rates. For example, at the false positive rate of 0.05 , the number of true positives detected by ALBERT pre-trained transformer-frozen model is almost 6 times more than that correctly predicted by Transformer or LSTM.

When the train/test set is split at random, i.e. there are similar proteins in the testing set to those in the training set, the performance of LSTM models is slightly better than the ALBERT pre-trained models, as shown in Table 1. However, the performance of the LSTM model significantly deteriorates when the proteins in the testing set are different from those in the training set. The ROC-AUC drops from over 0.9 to $\sim 0.4-0.6$, and PR-AUC decreases from over 0.8 to $~ 0.2-0.3$, while ALBERT pre-trained models could still maintain the ROC-AUC $\sim 0.7$ and the PR-AUC $\sim 0.5$. The significant drop in the performance for the prediction of dissimilar proteins is also observed in the Transformer model. These results suggest that the supervised learning alone is prone to overfitting, as observed in the Transformer and LSTM models, thus cannot be generalized to modeling remote orphan proteins. The pre-training of DISAE, which uses a large number of unlabeled proteins improve the generalization power. The training curves in Supplementary Figure 3, 4, 5 further support that ALBERT frozen transformer is generalizable, thus can reliably maintain its high performance when used for the deorphanization of dissimilar proteins. When evaluated by the dissimilar protein benchmark, the accuracy of training keeps increasing with the increased epochs; and the performance of ALBERT pre-trained transformer-frozen model is slightly worse than the most of models. However, the PR-AUC of ALBERT pre-trained transformer-frozen model is relatively stable and significantly higher than other models on the testing data. These observations further support that DISAE is capable of predicting the ligand binding to novel proteins.

\subsection{DISAE significantly outperforms state-of-the-art models for predicting ligand binding promiscuity across gene families}

We further evaluate the performance of ALBERT pre-trained model and compare it with the baseline Transformer and LSTM models on the prediction of ligand binding promiscuity across gene families. Specifically, we predict the binding of new kinase inhibitors to new GPCRs on the model that is trained using only GPCR sequences that are not known to bind to kinase inhibitors and chemicals that have not been annotated as kinase inhibitors. In other words, all GPCRs that bind to kinase inhibitors and all chemicals that are kinase inhibitors are excluded from the supervised training set. Although the kinase and the GPCRs belong to two completely different gene families in terms of sequence, structure, and function, a number of kinase inhibitors can bind to GPCRs as an off-target. We use these annotated GPCR-kinase inhibitor pairs as the test set. Interestingly, although DISAE is not extensively trained using a comprehensive chemical data set, the ALBERT pre-trained transformer-frozen model outperforms the LSTM and the Transformer (distilled triplets). As shown in Figure $3 \mathrm{C}$ and $3 \mathrm{D}$, both sensitivity and specificity of the ALBERT pre-trained transformer-frozen model outperforms other models. The ROC-AUC of ALBERT pre-trained transfomerfrozen model, Transformer, LSTM model is $0.690,0.687$, and 0.667 , respectively. Their PR-AUC is 0.673 , 0.630 , and 0.590 , respectively. The improvement is the most obvious in the region of low false positive ratio (the insert of Figure $3 \mathrm{C}$ ). At the false positive rate of 0.05 , the number of true positives detected by the ALBERT pre-trained transformer-frozen model is about $50 \%$ as many as that by Transfomer and LSTM models. This observation implies that the sequence pre-training captures certain ligand binding site information across gene families. It is noted that it is infeasible to apply homology modeling based method to infer ligand binding promiscuity across gene families.

\subsection{The effect of distilled sequence representation, pre-training, and fine- tuning}

To understand the contribution of each component in the ALBERT pre-training-fine-tuning procedure to the performance, we conduct a series of ablation studies as shown in Table 1 under the dissimilar protein and cross-gene-family benchmarks. For the three major pre-training-fine-tuning configurations of DISAE, distilled triplets sequence representation, pre-trained on whole Pfam, and fine-tuned with frozen transformer ("ALBERT frozen transformer" in Table 1), are recommended as the best performed model for predicting ligands of remote orphan proteins.

(a) Triplet is preferred over singlet on the ALBERT model

Under the same pre-training and fine-tuning settings, both ROC-AUC and PR-AUC of the ALBERT pre-trained model that uses the distilled triplets are significantly higher than the those when the distilled singlet is used. However, for LSTM model, the distilled singlet sometimes outperforms the distilled triplet. It could be because the triplet encodes more relational information on the remote proteins than close homologs.

(b) Pre-training on larger dataset is preferred

With the same fine-tuning strategy, frozen transformer, and use of distilled triplets, the model that is pre-trained on whole Pfam performs better than that pre-trained on the GPCR family alone in terms 
of both ROC-AUC and PR-AUC.

(c) Partial frozen transformer is preferred

With the same pre-training on whole Pfam and fine-tuning on the distilled triplets, the ALBERT pre-trained transformer-frozen model outperforms all other models that have only embedding layer frozen or both transformer and embedding layers frozen.

\subsection{DISAE learns biologically meaningful information}

Interpretation of deep learning is critical for its real-world applications. To understand if the trained DISAE model is biologically meaningful, we perform the model explainability analysis using SHapley Additive exPlanation (SHAP) [30. SHAP is a game theoretic approach to explain the output of any machine learning model. Shapley values could be interpreted as feature importance. We utilize this tool to get a closer look into the internal decision making of DISAE's prediction by calculating Shapley values of each triplet of a protein sequence. The average Shapley values of CPIs for a protein is used to highlight important positions for this protein.

Figure 4 shows the distribution of a number of residues of 5-hydroxytryptamine receptor $2 \mathrm{~B}$ on its structure, which are among $21(10 \%)$ residues with the highest SHAP values. Among them, 6 residues (T140, V208, M218, F341, L347, Y370) are located in the binding pocket. L378 is centered in the functional conserved NPxxY motif that connects the transmembrane helix 7 and the cytoplasmic helix 8 and plays a critical role in the activation of GPCRs 31]32. P160 and I161 is the part of intracellular loop 2, while I192, G194, I195, and E196 are located in the extracellular loop 2. The intracellular loop 2 interacts with the P-loop of G-protein [33. It is proposed that the extracellular loop 2 may play a role in the selective switch of ligand binding and determine ligand binding selectivity and efficacy 34 35 36] 37. The functional impact of other residues are unclear. Nevertheless, more than one half of top 21 residues ranked by SHAP values can explain the trained model. The enrichment of ligand binding site residues is statistically significant $(\mathrm{p}$-value $=0.01)$. These results suggest that the prediction from DISAE can provide biologically meaningful interpretations.

\subsection{Application to the hierarchical classification and deorphanization of human GPCRs}

With the established generalization power of DISAE, we use ALBERT transformer-frozen model pretrained on whole Pfam in distilled triplets form to tackle the challenge of deorphanization of human GPCRs, due to its consistently excellent performance by all evaluation metrics in the benchmarks.

We define the orphan GPCRs as those which do not have known small molecule binders. 649 human GPCRs that are annotated in Pfam families PF00001, PF13853, and PF03402 are identified as the orphan receptors.

Studies have suggested that the classification of GPCRs should be inferred by combining sequence and ligand information [38. The protein embedding of the DISAE model after pre-training-fine-tuning satisfies this requirement. Therefore, we use the cosine similarity between the embedded vector of protein as a metric to cluster the human GPCRome, which includes both non-orphan and orphan GPCRs. The hierarchical clustering of GPCRs in the Pfam PF00001 is shown in Figure 5 Supplementary Figure 6 and 7 show the hierarchical clustering of PF13853 and PF03402, respectively.

Table 2 provides examples of predicted approved-drug bindings to the orphan GPCRs by DISASE with high confidence (false positive rate $<5.0 \mathrm{e}-4$ ). The complete list of orphans human GPCRs paired with 555 approved GPCR-targeted drugs is in the Supplementary Table 1. The predicted potential interactions between the approved drug and the orphan receptor will not only facilitate designing experiments to deorphanize GPCRs but also provide new insights into the mode of action of existing drugs for drug repurposing, polypharmacology, and side effect prediction.

\section{Conclusion}

Our primary goal in this paper is to address the challenge of predicting ligands of orphan proteins that are significantly dissimilar from proteins that have known ligands or solved structures. To address this challenge, we introduce new techniques for the protein sequence representation by the pre-training of distilled sequence alignments, and the module-based fine-tuning using labeled data. Our approach, DISAE, is inspired by the state-of-the-art algorithms in NLP. However, our results suggest that the direct adaption of NLP may be less fruitful. The successful application of NLP to biological problems requires the incorporation of domain knowledge in both pre-training and fine-tuning stages. In this regard, DISAE significantly improves the state-of-the-art in the deorphanization of dissimilar orphan proteins. Nevertheless, DISAE can be further improved in several aspects. First, more biological knowledge can be incorporated into the pre-training and fine-tuning at both molecular level (e.g. protein structure and ligand binding site information) and system level (e.g. protein-protein interaction network). Second, in the framework of self-supervised learning, a wide array of techniques can be adapted to address the problem of bias, sparsity, and noisiness in the training 
data. Put together, new machine learning algorithms that can predict endogenous or surrogate ligands of orphan proteins open up a new avenue for deciphering biological systems, drug discovery, and precision medicine.

\section{Methods}

\subsection{Protein sentence representation from distilled sequence alignment}

A protein sequence is converted into an ordered list of amino acid fragments (words) with the following steps, as illustrated in Figure 1 B .

1. Given a sequence of interest $S$, a multiple sequence alignment is constructed by a group of similar sequences to $S$. In this paper, the pre-computed alignments in Pfam [22] are used.

2. Amino acid conservation at each position is determined.

3. Starting from the most conserved positions that are defined in the Pfam, a pre-defined number of positions in the alignment are selected by the ranking of conservation. In this study, the number of positions is set as 210. The length of positions was not optimized.

4. A word, either a single amino acid or a triplet of amino acids in the sequence, is selected at each position. The triplet may include gaps.

5. Finally, all selected words from the sequence form an ordered list following the sequence order, i.e. the sentence representation of the protein.

The rationale for the distilled multiple sequence alignment representation is to only use functionally or evolutionarily important residues and ignore others. It can be considered a feature selection step. In addition, the use of multiple sequence alignment will allow us to correlate the functional information with the position encoding. The distilled sequence will not only reduce the noise but also increase the efficiency in the model training since the memory and time complexity of language model is $O\left(n^{2}\right)$. It is noted that we use the conservation to select residues in a sequence because it is relevant to protein function and ligand binding. Other criteria (e.g., co-evolution) could be used depending on the down-stream applications (e.g., protein structure prediction).

\subsection{Distilled sequence alignment embedding (DISAE) using ALBERT}

It has been shown that Natural Language Processing (NLP) algorithms can be successfully used to extract biochemically meaningful vectors by pre-training BERT [29] on 86 billion amino acids (words) in 250 million protein sequences 39]. A recently released light version of BERT, called ALBERT [17, boasts significantly lighter memory uses with better or equivalent performances compared to BERT [29]. We extend the idea of unsupervised pre-training of proteins using ALBERT algorithm using distilled multiple sequence alignment representation. The distilled ordered list of triplets is used as the input for ALBERT pre-training. In this work, only masked language model (MLM) is used for the pre-training.

\subsection{Architecture of deep learning model for whole-genome CPI prediction}

The deep learning model for the whole-genome CPI prediction is mainly composed of three components, as shown in Figure 1 C, protein embedding by DISAE, chemical compound embedding, and attention pooling with multilayer perceptron (MLP) to model CPIs. DISAE is described in the previous section. Note that once processed through ALBERT, each protein is represented as a matrix of size 210 by 312 , where each triplet is a vector of length 312. During protein-ligand interaction prediction task, the protein embedding matrix is compressed using ResNet [40. Once processed through ResNet layers, each protein is represented as a vector of length 256, which contains compressed information for the whole 210 input triplets for the corresponding protein.

Neural molecular fingerprint [4] is used for the chemical embedding. Small molecule is represented as a $2 \mathrm{D}$ graph, where vertices are atoms and edges are bonds. We use a popular graph convolutional neural network to process ligand molecules 27 .

The attentive pooling is similar to the design in 11. For each putative chemical-protein pair, the corresponding embedding vectors are fed to the attentive pooling layer, which in turn produces the interaction vector.

More details on the neural network model configurations can be found in Supplementary Table 2, 3, 4 .

\subsection{Module-based fine tuning strategy}

When applying ALBERT to a supervised learning task, fine-tuning [17] is a critical step for the task-specific training following the pre-training. Pre-trained ALBERT already learned to generate protein representation in a meaningful way. However, it is also a design choice whether to allow ALBERT to get updated and trained together with the other components of the classification system during the fine-tuning [17, 42]. Updating ALBERT during the fine-tuning will allow the pre-trained protein encoder to better capture knowledge from the training data while minimizing the risk of significant loss of knowledge obtained from 
the pre-training. Hence, to find the right balance, we experiment with ALBERT models that are partially unfrozen 42. To be specific, major modules in the ALBERT model, embedding or transformer 43, are unfrozen separately as different variants of the model. The idea of "unfrozen" layers is widely used in Natural Language Processing (NLP), e.g., ULMFiT [42] where model consists of several layers. As training proceeds, layers are gradually unfrozen to learn the task-specific knowledge while safeguarding knowledge gained from pre-training. However, this is not straightforwardly applicable to ALBERT because ALBERT is not a linearly layered-up architecture. Hence, we apply a module-based unfrozen strategy.

\subsection{Experiments Design}

The purpose of this study is to build a model to predict chemical binding to novel orphan proteins. Therefore, we design experiments to examine the model generalization capability to the data not only unseen but also from significantly dissimilar proteins. We split training/validation/testing data sets to assess the performance of algorithms in three scenarios: 1 . The proteins in the testing data set are significantly different from those in the training and validation data set based on the sequence similarity. 2. The ligands in the testing data are from a different gene family from that in the training/validation data. 3. Whole data set is randomly split similar to most of the existing work.

To examine the effect of different pre-training-fine-tuning algorithms, we organize experiments in three categories of comparison, as shown in Table 1

(a) THE EFFECT OF VOCABULARY: Taking protein sequence as a sentence, its vocabulary could be built in many ways. We compare taking the singlet as vocabulary against the triplet as vocabulary.

(b) THE EFFECT OF PRE-TRAINING: We assess how unlabeled protein sequences affect the performance of the classification. We compare ALBERT pre-trainined on whole Pfam alone against one pretrainined on GPCRs alone and one without pre-training.

(c) THE EFFECT OF FINE-TUNING: We compare three ALBERT models: ALBERT all unfrozen, ALBERT frozen embedding, and ALBERT frozen transformer [4]. All of these modes are pre-trained on the whole Pfam.

We use LSTM [44] and Transformer (distilled triplets) as baselines. Two variants of LSTM models are tested to compare with the above three groups of experiments: LSTM with distilled triplets and distilled singlets.

\subsection{Dataset}

Our task is to learn from large-scale CPI data to predict unexplored interactions. The quality and quantity of the training samples are critical for biologically meaningful predictions. Despite continuous efforts in the community, a single data source typically curates an incomplete list of our knowledge in protein-ligand activities. Thus, we integrated multiple high-quality, large-scale, publicly available databases of known protein-ligand activities. We extracted, split, and represented protein-ligand activity samples for training and evaluation of machine learning-based predictions.

- Sequence data for ALBERT pre-training

Proteins sequences are first collected from Pfam-A 22] database. Then, sequences are clustered by $90 \%$ sequence identity, and a representative sequence is selected from each cluster. The 40,282,439 sequences (including 138,288 GPCR) are used for the ALBERT pre-training. To construct protein sentences from the distilled sequence alignment, the original alignment and conservation score from each Pfam-A family are used. As a comparison, the 35,181 GPCR sequences only from GPCR family PF00001 are used to pre-train a separated ALBERT model.

We used Pfam alignments directly. From the consensus alignment sequence, we collected positions of high-confidence and low-confidence conserved amino acids together with conservatively substituted ones. We picked these positions from each of the target GPCRs. As a result, each GPCR is represented by 210 amino acids, which may contain gaps. The 210 amino acids are then treated as a sentence of triplets. The triplets are used to train the ALBERT model with the following parameters: maximum sequence length $=256$, maximum predictions per sentence $=40$, word masking probability $=0.15$, and duplication factor $=10$. Note that the order of different GPCRs in multiple sequence alignment may not be biologically meaningful. Thus, we did not apply the next sentence prediction task during the pre-training.

- Binding assay data for supervised learning

We integrated protein-ligand activities involving any GPCRs from ChEMBL [23] (ver. 25), BindingDB 24] (downloaded Jan 9, 2019), GLASS [25] (downloaded Nov 26, 2019), and DrugBank [26] (ver. 5.1.4). Note that BindingDB also contains samples drawn from multiple sources, including PubChem, PDSP $K_{i}$, and U.S. Patent. From ChEMBL, BindingDB, and GLASS databases, protein-ligand activity assays measured in three different unit types, $p K_{d}, p K_{i}$, and $p I C_{50}$ are collected. Log-transformation were performed for activities reported in $K_{d}, K_{i}$, or $I C_{50}$. For consistency, we did not convert different activity types. For instance, activities reported in $I C_{50}$ are converted only to $p I_{50}$, but not any other activity types. The activities in log-scale were then binarized based on the thresholds of activity values. 
Protein-ligand pairs were considered active if $p I C_{50}>5.3$, or $p K_{d}>7.3$ or $p K_{i}>7.3$, and inactive if $p I C_{50}<5.0, p K_{d}<7.0$ or $p K_{i}<7.0$, respectively.

$$
\begin{aligned}
p I C_{50} & =-\log \left(I C_{50}\right) \\
p K_{i} & =-\log \left(K_{i}\right) \\
p K_{d} & =-\log \left(K_{d}\right)
\end{aligned}
$$$$
Y \in R^{m \times n}
$$

$$
Y_{i, j}=1 \text { if }\left\{\begin{array}{l}
\frac{\sum_{k=1}^{\left|A_{1}(i, j)\right|} A_{1}(i, j)_{k}}{\left|A_{1}(i, j)\right|} \geq 5.3 \\
\frac{\sum_{k=1}^{\left|A_{2}(i, j)\right|} A_{2}(i, j)_{k}}{\left|A_{2}(i, j)\right|} \geq 7.3 \\
\frac{\sum_{k=1}^{\left|A_{3}(i, j)\right|} A_{3}(i, j)_{k}}{\left|A_{3}(i, j)\right|} \geq 7.3
\end{array}\right.
$$

$$
Y_{i, j}=-1 \text { if }\left\{\begin{array}{l}
\frac{\sum_{k=1}^{\left|A_{1}(i, j)\right|} A_{1}(i, j)_{k}}{\left|A_{1}(i, j)\right|} \leq 5.0 \\
\frac{\sum_{k=1}^{\left|A_{2}(i, j)\right|} A_{2}(i, j)_{k}}{\left|A_{2}(i, j)\right|} \leq 7.0 \\
\frac{\sum_{k=1}^{\left|A_{3}(i, j)\right|} A_{3}(i, j)_{k}}{\left|A_{3}(i, j)\right|} \leq 7.0
\end{array}\right.
$$

In the above equations, $m$ and $n$ are the total number of unique proteins and ligands, respectively. $A_{1}(i, j), A_{2}(i, j)$, and $A_{3}(i, j)$ are the list of all activity values for the $i$ th protein and $j$ th ligand in $p I C_{50}, p K_{d}$, and $p K_{i}$, respectively. $|A|$ denotes the cardinality of the set $A$. Note that there are gray areas in the activity thresholds. Protein-ligand pairs falling in the gray areas were considered undetermined and unused for training. If multiple activities were reported for a protein-ligand pair, their log-scaled activity values were averaged for each activity type and binarized accordingly. In addition, we collected active protein-ligand associations from DrugBank and integrated with the binarized activities mentioned above. Inconsistent activities (e.g. protein-ligand pairs that appear both active and inactive) were removed. There are total 9705 active and 25175 inactive pairs, respectively, in the benchmark set.

\subsection{Benchmark}

To test the model generalization capability, a protein similarity based data splitting strategy is implemented. First, pair-wise protein similarity based on bit-score is calculated using BLAST [45] for all GPCRs in the data set. The similarity between $\operatorname{protein}_{i}$ and $\operatorname{protein}_{j}$ is define as:

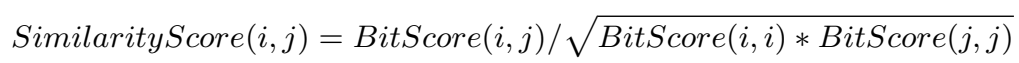

Then, according to the similarity distribution, a similarity threshold is set for the splitting. The bit-score similarity threshold is 0.035 . The sequences are clustered such that the sequences in the testing set are significantly dissimilar from those in the training/validation set, as shown in 2 After the splitting, there are 25114, 6278, and 3488 samples for training, validation, and testing, respectively. Distribution of protein sequence similarity scores can be found in Supplementary Figure 8.

\subsection{Ensemble model for the GPCR deorphanization}

All annotated GPCR-ligand binding pairs are used to build prediction model for the GPCR deorphanization. To reduce possible overfitting, an ensemble model is constructed using the chosen ALBERT model. Following the strategy of cross-validation [46], three DISAE models are trained. Similar to the benchmark experiments, a hold-out set is selected based on protein similarity, and is used for the early stopping at the preferred epoch for each individual model. Max-voting [4] is invoked to make final prediction for the orphan human GPCRs.

To estimate the false positive rate of predictions on the orphan-chemical pairs, a prediction score distribution is collected for known positive pairs and negative pairs of testing data. Assuming that the prediction score of orphan pairs have the same distribution as that of the testing data, for each prediction score, a false positive rate can be estimated based on the score distribution of true positives and negatives.

\subsection{SHAP analysis}

Kernel SHAP [30] is used to calculate SHAP values for distilled protein sequences. It is a specially-weighted local linear regression to estimate SHAP values for any model. The model used is the classifier that finetunes the ALBERT frozen transformer, and is pre-trained on whole Pfam in distilled triplets under the remote GPCR setting. The data used is the testing set generated under the same remote GRPC setting. Although the whole input features to the classifier consist of both distilled protein sequence and chemical neural fingerprint, only protein feature is of the interest. Hence, when calculating the base value (a value that would be predicted if we do not know any features) required by SHAP analysis, all testing set protein sequence are masked with the same token, while chemical neural fingerprint remain untouched. Therefore, the base value is the average prediction score without protein feature and solely relying on chemical feature. Since the distilled sequences are all set to be of length 210, the SHAP values are the feature importance for each of the 210 positions. 


\subsection{Hierarchical clustering of the human GPCRome}

The pairwise distance between two proteins is determined by the cosine similarity of the DISAE embedding vector after pre-training-fine-tuning, which incoporates both sequence and ligand information. $R$ pacakge "ape" (Analyses of Phylogenetics and Evolution) 48 and treeio 49 is used to convert a distance matrix of proteins into a Newick tree format. Gtree [50] [51] package is used to plot the tree in a circular layout.

\section{References}

[1] Oprea, T. Exploring the dark genome: implications for precision medicine. Mammalian Genome 30 (2019).

[2] Rodgers, G. et al. Glimmers in illuminating the druggable genome. Nature Reviews Drug Discovery $\mathbf{1 7}$ (2018).

[3] Laschet, C., Dupuis, N. \& Hanson, J. The g protein-coupled receptors deorphanization landscape. Biochemical Pharmacology 153 (2018).

[4] Ngo, T. et al. Identifying ligands at orphan gpcrs: Current status using structure-based approaches. British Journal of Pharmacology 173, n/a-n/a (2016).

[5] Zheng, S., Li, Y., Chen, S., Xu, J. \& Yang, Y. Predicting drugâ"protein interaction using quasi-visual question answering system. Nature Machine Intelligence 2, 134-140 (2020).

[6] Lewis, T. E. et al. Genome3D: exploiting structure to help users understand their sequences. Nucleic Acids Research 43, D382-D386 (2014). URL https://doi.org/10.1093/nar/gku973. https: //academic.oup.com/nar/article-pdf/43/D1/D382/7330713/gku973.pdf

[7] Cheng, Z. et al. Effectively identifying compound-protein interactions by learning from positive and unlabeled examples. IEEE/ACM Transactions on Computational Biology and Bioinformatics PP, 1-1 (2016).

[8] Lim, H. et al. Large-scale off-target identification using fast and accurate dual regularized one-class collaborative filtering and its application to drug repurposing. PLOS Comput Biol 12 (2016).

[9] Lim, H., Gray, P., Xie, L. \& Poleksic, A. Improved genome-scale multi-target virtual screening via a novel collaborative filtering approach to cold-start problem. Scientific Reports 6, 38860 (2016).

[10] Wen, M. et al. Deep-learning-based drug-target interaction prediction. Journal of Proteome Research 16 (2017).

[11] Gao, K. Y. et al. Interpretable drug target prediction using deep neural representation. In Proceedings of the Twenty-Seventh International Joint Conference on Artificial Intelligence, IJCAI-18, 3371-3377

(International Joint Conferences on Artificial Intelligence Organization, 2018). URL https://doi .org/ 10.24963/ijcai.2018/468

[12] Nguyen, T., Le, H. \& Venkatesh, S. Graphdta: prediction of drug-target binding affinity using graph convolutional networks (2019).

[13] Lee, I., Keum, J. \& Nam, H. Deepconv-dti: Prediction of drug-target interactions via deep learning with convolution on protein sequences. PLOS Computational Biology 15, e1007129 (2019).

[14] Sutskever, I., Vinyals, O. \& Le, Q. Sequence to sequence learning with neural networks. 10 (2014).

[15] Karimi, M., Wu, D., Wang, Z. \& Shen, Y. Deepaffinity: Interpretable deep learning of compoundprotein affinity through unified recurrent and convolutional neural networks (2018).

[16] Chen, L. et al. Transformercpi: Improving compound-protein interaction prediction by sequence-based deep learning with self-attention mechanism and label reversal experiments. Bioinformatics (Oxford, England) (2020).

[17] Lan, Z. et al. Albert: A lite bert for self-supervised learning of language representations (2019).

[18] Rao, R. et al. Evaluating protein transfer learning with TAPE. CoRR abs/1906.08230 (2019). URL http://arxiv.org/abs/1906.08230, 1906.08230.

[19] Bepler, T. \& Berger, B. Learning protein sequence embeddings using information from structure. CoRR abs/1902.08661 (2019). URL http://arxiv.org/abs/1902.08661 1902.08661.

[20] Min, S., Park, S., Kim, S., Choi, H.-S. \& Yoon, S. Pre-training of deep bidirectional protein sequence representations with structural information (2019).

[21] Hauser, A., Attwood, M. M., Rask-Andersen, M., Schi $\Upsilon$ th, H. \& Gloriam, D. Trends in gpcr drug discovery: New agents, targets and indications. Nature Reviews Drug Discovery 16, nrd.2017.178 (2017).

[22] El-Gebali, S. et al. The pfam protein families database in 2019. Nucleic acids research 47 (2018).

[23] Gaulton, A. et al. Chembl: a large-scale bioactivity database for drug discovery. Nucleic acids research 40, D1100-7 (2011). 
[24] Lin, Y., Liu, M. \& Gilson, M. The binding database: data management and interface design. Bioinformatics (Oxford, England) 18, 130-9 (2002).

[25] Chan, W. et al. Glass: A comprehensive database for experimentally validated gpcr-ligand associations. Bioinformatics (2015).

[26] Wishart, D. et al. Drugbank: A comprehensive resource for in silico drug discovery and exploration. Nucleic acids research 34, D668-72 (2006).

[27] Duvenaud, D. K. et al. Convolutional networks on graphs for learning molecular fingerprints 2224-2232 (2015). URL http://papers.nips.cc/paper/ 5954-convolutional-networks-on-graphs-for-learning-molecular-fingerprints.pdf

[28] dos Santos, C. N., Tan, M., Xiang, B. \& Zhou, B. Attentive pooling networks. CoRR abs/1602.03609 (2016). URL http://arxiv.org/abs/1602.03609 1602.03609

[29] Devlin, J., Chang, M.-W., Lee, K. \& Toutanova, K. BERT: Pre-training of deep bidirectional transformers for language understanding. In Proceedings of the 2019 Conference of the North American Chapter of the Association for Computational Linguistics: Human Language Technologies, Volume 1 (Long and Short Papers), 4171-4186 (Association for Computational Linguistics, Minneapolis, Minnesota, 2019). URL https://www.aclweb.org/anthology/N19-1423

[30] Lundberg, S. M. \& Lee, S.-I. A unified approach to interpreting model predictions. In Guyon, I. et al. (eds.) Advances in Neural Information Processing Systems 30, 4765-4774 (Curran Associates, Inc., 2017). URL http://papers.nips.cc/paper/ 7062-a-unified-approach-to-interpreting-model-predictions.pdf

[31] Fritze, O. et al. Role of the conserved npxxy(x)5,6f motif in the rhodopsin ground state and during activation. Proceedings of the National Academy of Sciences of the United States of America 100, 2290-5 (2003).

[32] Trzaskowski, B. et al. Action of molecular switches in gpcrs - theoretical and experimental studies. Current medicinal chemistry 19, 1090-109 (2012).

[33] Hilger, D., Masureel, M. \& Kobilka, B. Structure and dynamics of gpcr signaling complexes. Nature Structural Molecular Biology 25 (2018).

[34] Woolley, M. \& Conner, A. Understanding the common themes and diverse roles of the second extracellular loop (ecl2) of the gpcr super-family. Molecular and Cellular Endocrinology 449 (2016).

[35] Peeters, M., Van Westen, G., Li, Q. \& IJzerman, A. Importance of the extracellular loops in g proteincoupled receptors for ligand recognition and receptor activation. Trends in pharmacological sciences 32, 35-42 (2010).

[36] Seibt, B. et al. The second extracellular loop of gpcrs determines subtype-selectivity and controls efficacy as evidenced by loop exchange study at a2 adenosine receptors. Biochemical pharmacology $\mathbf{8 5}$ (2013).

[37] Perez-Aguilar, J. M., Shan, J., LeVine, M., Khelashvili, G. \& Weinstein, H. A functional selectivity mechanism at the serotonin-2a gpcr involves ligand-dependent conformations of intracellular loop 2. Journal of the American Chemical Society 136 (2014).

[38] Scholz, N., Langenhan, T. \& Schöneberg, T. Revisiting the classification of adhesion gpcrs. Annals of the New York Academy of Sciences 1456, 80 (2019).

[39] Rives, A. et al. Biological structure and function emerge from scaling unsupervised learning to 250 million protein sequences (2019).

[40] He, K., Zhang, X., Ren, S. \& Sun, J. Deep residual learning for image recognition. CoRR abs/1512.03385 (2015). URL http://arxiv.org/abs/1512.03385 1512.03385.

[41] Duvenaud, D. K. et al. Convolutional networks on graphs for learning molecular fingerprints 2224-2232 (2015). URL http://papers.nips.cc/paper/ 5954-convolutional-networks-on-graphs-for-learning-molecular-fingerprints.pdf.

[42] Howard, J. \& Ruder, S. Universal language model fine-tuning for text classification. 328-339 (2018).

[43] Vaswani, A. et al. Attention is all you need (2017).

[44] Van Houdt, G., Mosquera, C. \& NÃ ipoles, G. A review on the long short-term memory model. Artificial Intelligence Review (2020).

[45] Altschul, S. et al. Gapped blast and psi-blast: a new generation of protein databases search programs. Nucleic acids research 25, 3389-402 (1997).

[46] Kohavi, R. A study of cross-validation and bootstrap for accuracy estimation and model selection 14 (2001).

[47] Leon, F., Floria, S.-A. \& Badica, C. Evaluating the effect of voting methods on ensemble-based classification. 1-6 (2017). 
[48] Paradis, E. \& Schliep, K. ape 5.0: an environment for modern phylogenetics and evolutionary analyses in R. Bioinformatics 35, 526-528 (2019).

[49] Wang, L.-G. et al. treeio: an r package for phylogenetic tree input and output with richly annotated and associated data. Molecular Biology and Evolution accepted (2019).

[50] Yu, G., Smith, D., Zhu, H., Guan, Y. \& Lam, T. T.-Y. ggtree: an r package for visualization and annotation of phylogenetic trees with their covariates and other associated data. Methods in Ecology and Evolution 8(1):28-36 (2017).

[51] Yu, G., Lam, T. T.-Y., Zhu, H. \& Guan, Y. Two methods for mapping and visualizing associated data on phylogeny using ggtree. Methods in Ecology and Evolution 35(2):3041-3043 (2018).

\section{Acknowledgement}

This project has been funded in whole or in part with federal funds from the National Institute of General Medical Sciences of National Institute of Health (R01GM122845), the National Institute on Aging of the National Institute of Health (R01AD057555), and the National Cancer Institute of National Institutes of Health, under contract HHSN261200800001E. The content of this publication does not necessarily reflect the views or policies of the Department of Health and Human Services, nor does mention of trade names, commercial products or organizations imply endorsement by the US Government. This Research was supported [in part] by the Intramural Research Program of the NIH, National Cancer Institute, Center for Cancer Research and the Intramural Research Program of the NIH Clinical Center.

\section{Author Contributions}

LX conceived and planned the experiments. TC and HL developed and implemented the algorithm. TC, HL, KAA, and YQ carried out the experiments. LX, TC, HL, and KAA contributed to the interpretation of the results. TC, HL, KAA, RN, and LX wrote the manuscript. All authors provided critical feedback and helped shape the research, analysis and manuscript.

\section{Competing Interests Statement}

We declare that we have no financial and personal relationships with other people or organizations that can inappropriately influence our work, there is no professional or other personal interest of any nature or kind in any product, service and/or company that could be construed as influencing the position presented in, or the review of, the manuscript entitled. 
bioRxiv preprint doi: https://doi.org/10.1101/2020.10.08.332346; this version posted October 9, 2020. The copyright holder for this preprint (which was not certified by peer review) is the author/funder. All rights reserved. No reuse allowed without permission.

${ }_{553}$ Figures and Tables 
A

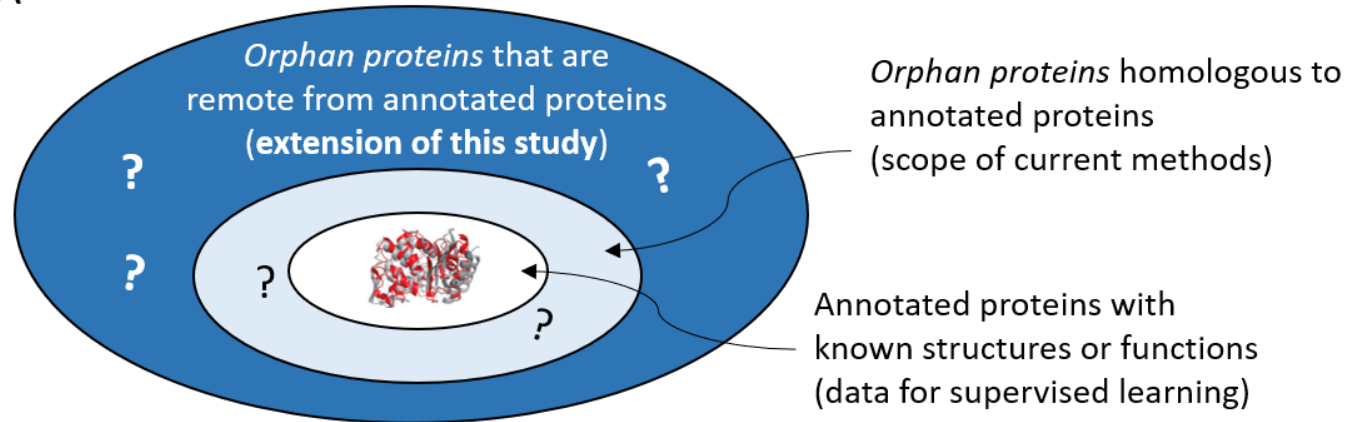

B

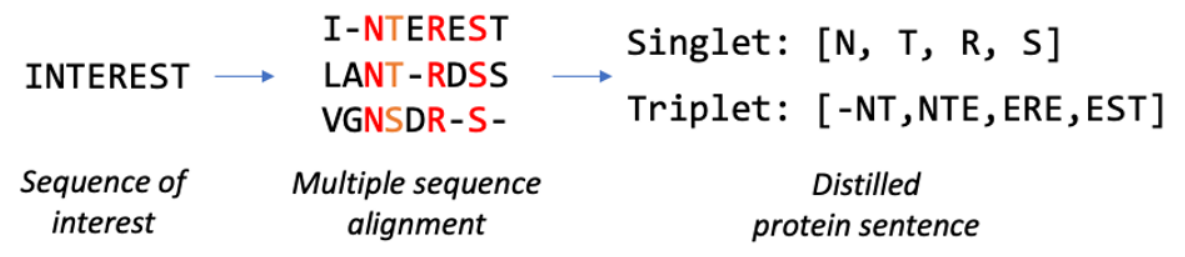

C

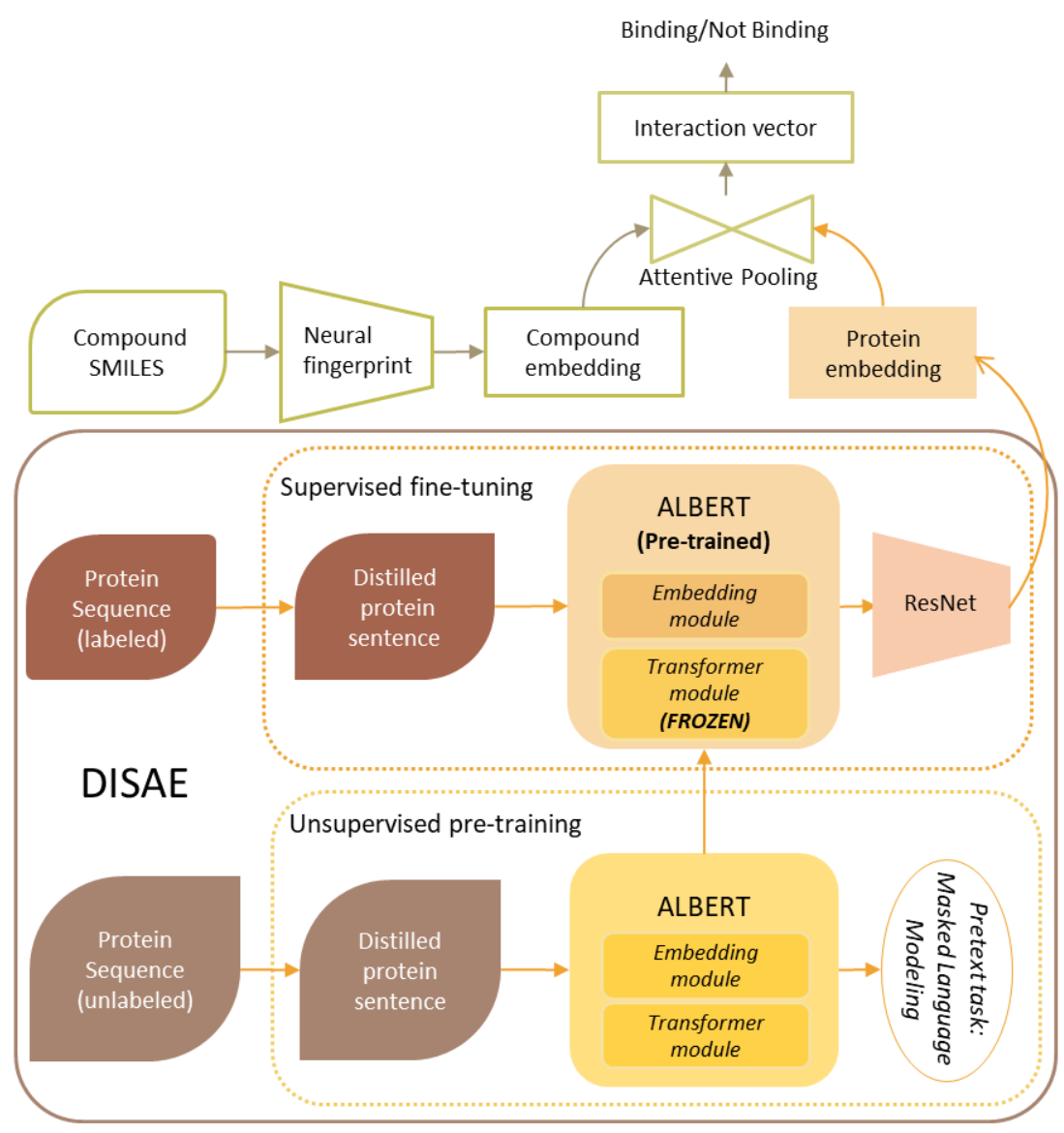

Figure 1: (A) Comparison of the scope of this study with that of current works. In existing methods, only annotated proteins (labeled data) are used in the model training. They work well when an orphan protein is homologous to the annotated protein, but mostly fail when the orphan protein is dissimilar from the annotated protein. By contrast, this study uses both annotated and orphan proteins in a self-supervised-learning-fine-tuning framework, thus extends the scope to genome-wide remote orphan proteins that are out of the reach of state-of-the-arts. (B) Illustration of protein sequence representation from distilled sequence alignment. The high and medium conserved positions are marked as red and orange, respectively. (C) Architecture of deep learning model for the wholegenome CPI prediction 


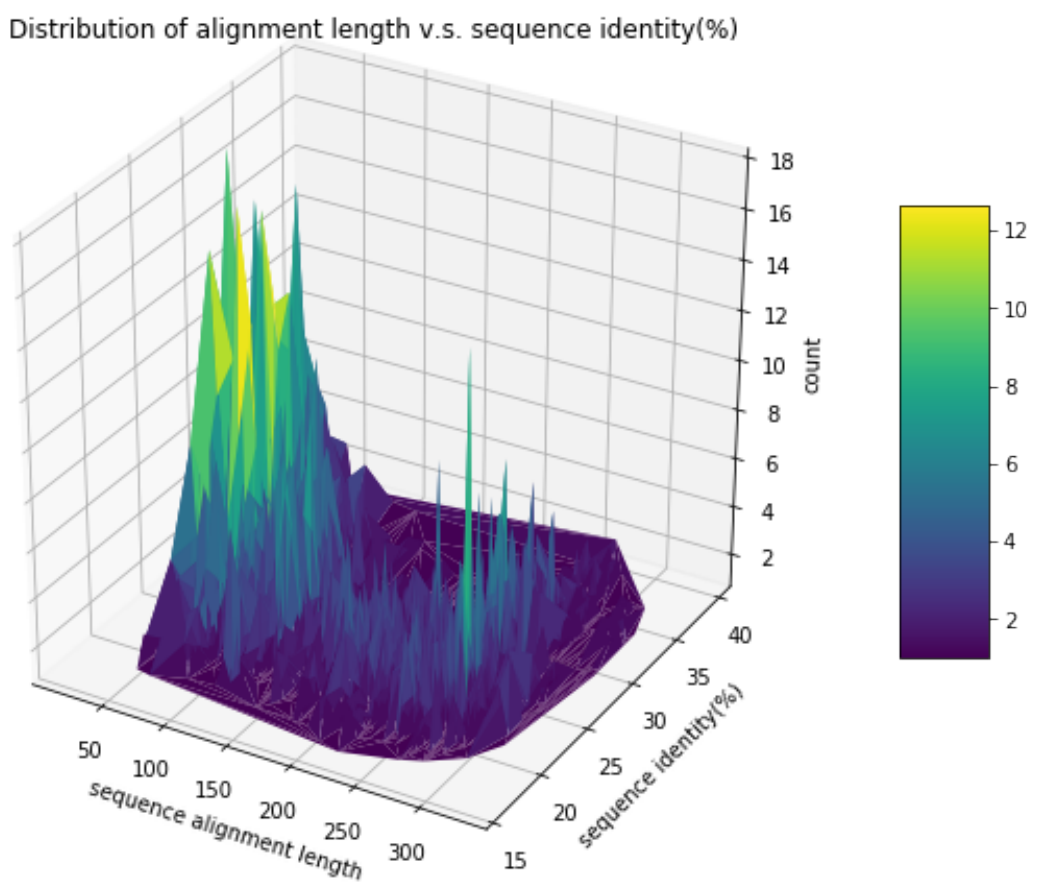

Figure 2: Distribution of sequence alignment length and percentage of sequence identity between proteins in the testing set (query) and those in the training/validation set (template) in the dissimilar protein benchmark. The sequence length of each protein is around 300. 


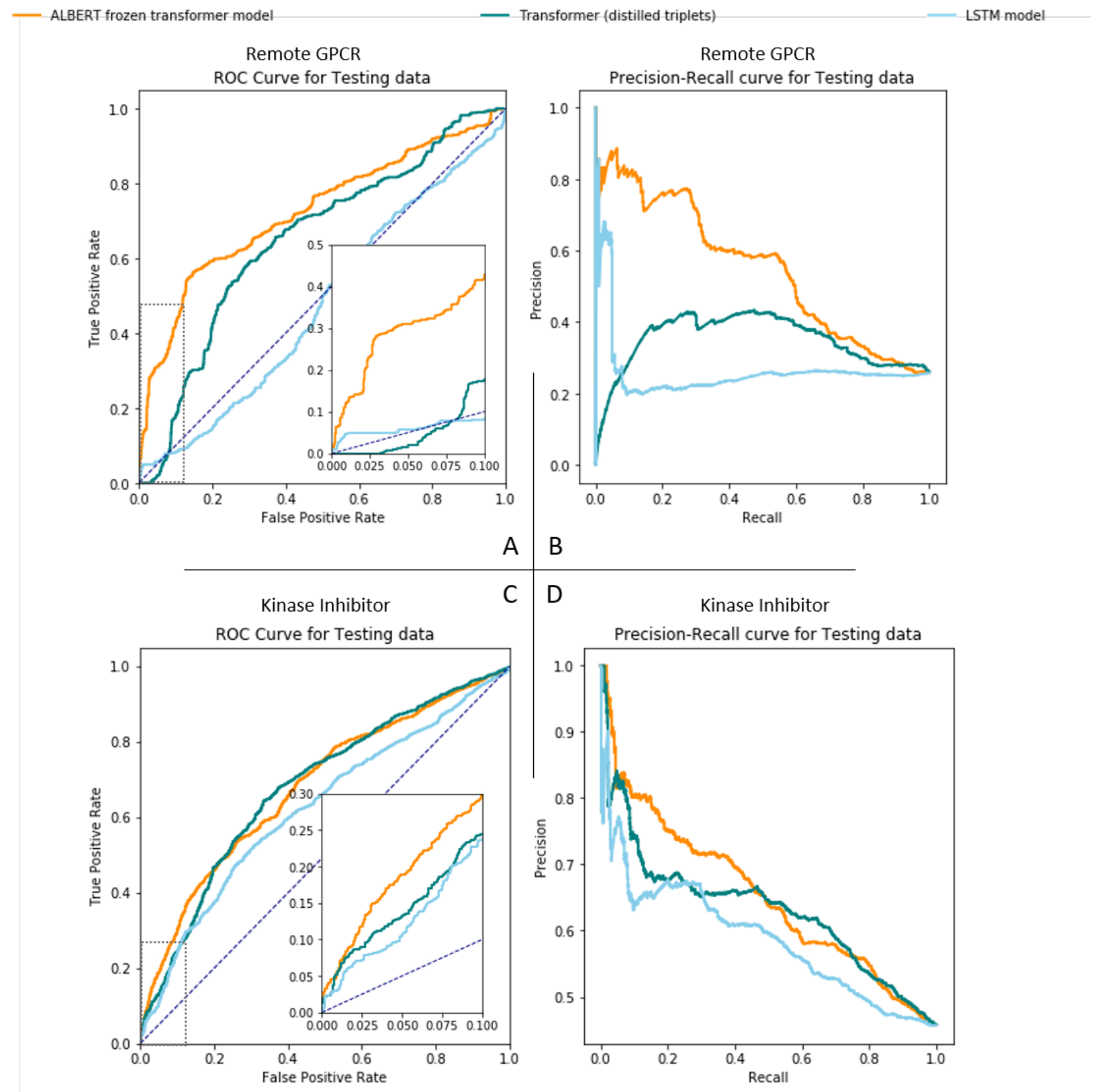

Figure 3: Performance comparison of ALBERT pre-trained transformer-frozen model, Transformer model, and LSTM model. (A, B): ROC- and PR-curves for the prediction of ligand binding to remote GPCRs (more than $90 \%$ between any query proteins and any template proteins). (C, D): ROC- and PR-curves for the classification on testing set in the cross-gene-family kinase inhibitor benchmark. All the models are trained on distilled triplets. 


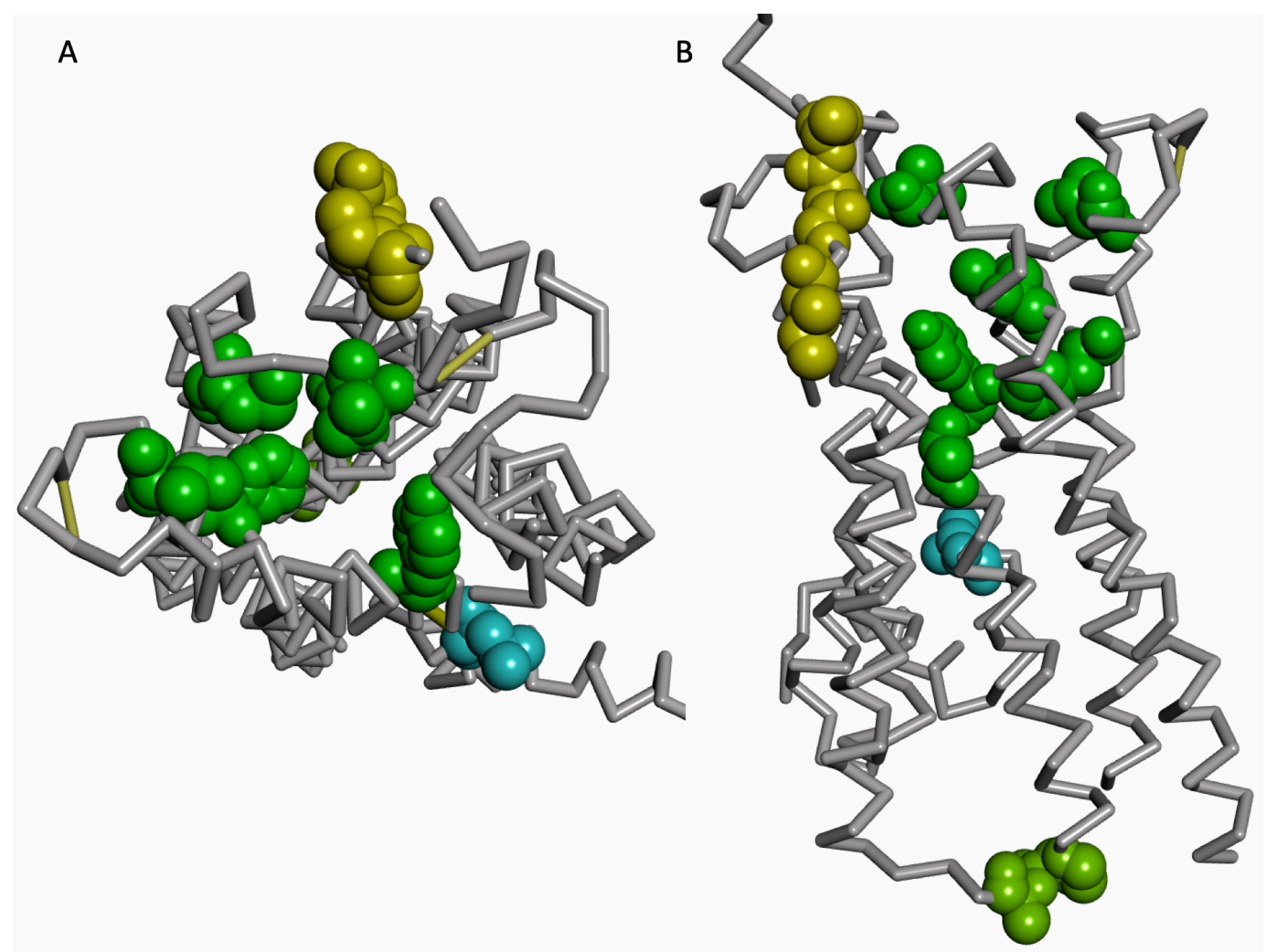

Figure 4: (A) top and (B) side view of structure of 5-hydroxytryptamine receptor 2B ( UNIPROT id: 5HTB2_HUMAN, PDB ID: 4IB4). The residues among those with the top 21 ranked SHAP values are shown in dark green, blue, yellow, and light green colored CPK mode for the amino acids in the binding pocket, NPxxY motif, extracellular loop, and intracellular loop, respectively. 


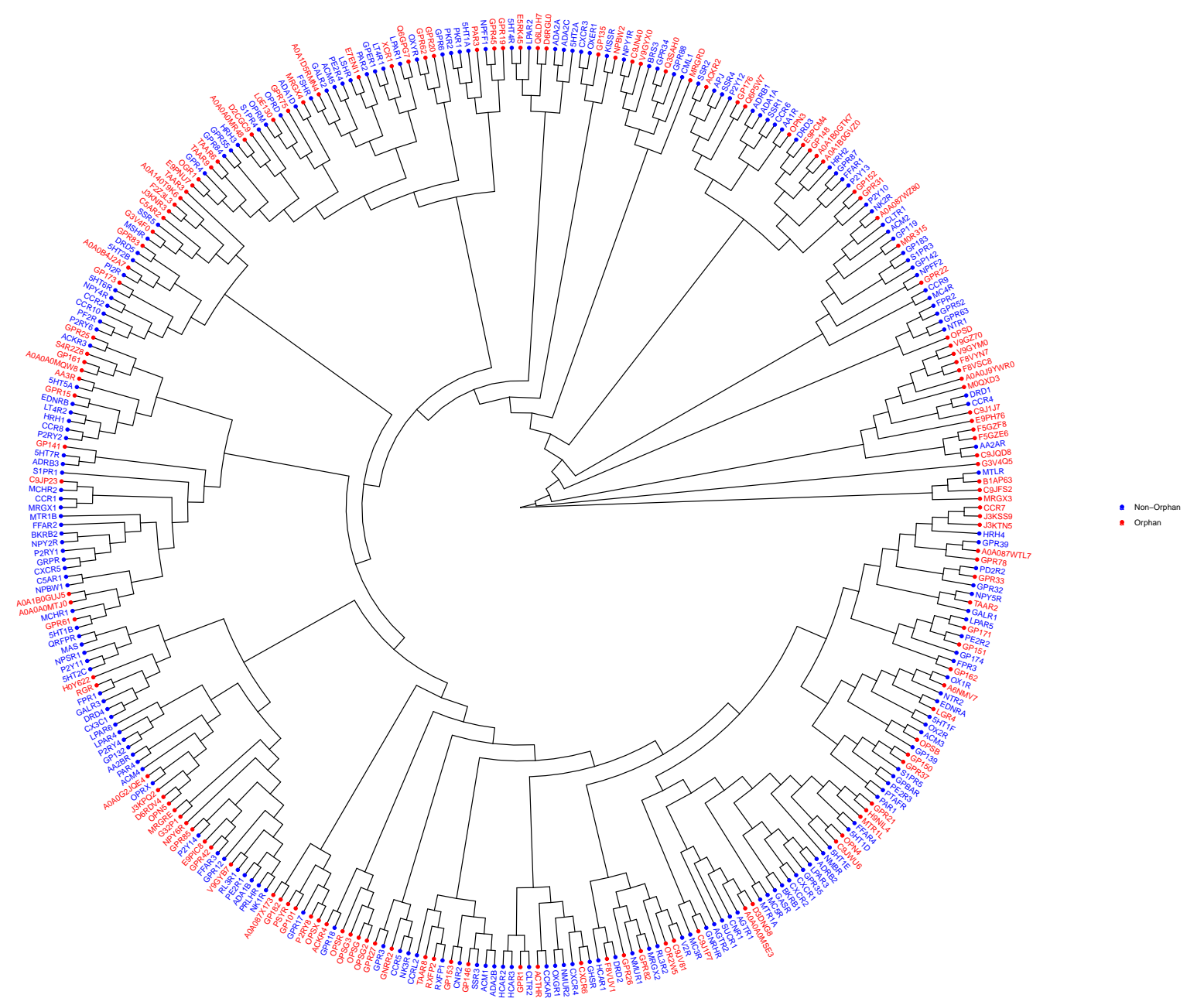

Figure 5: Hierarchical clustering of human GPCRs in the Pfam PF00001. Non-orphan and orphan GPCRs are labeled in blue and red, respectively 
bioRxiv preprint doi: https://doi.org/10.1101/2020.10.08.332346; this version posted October 9, 2020. The copyright holder for this preprint (which was not certified by peer review) is the author/funder. All rights reserved. No reuse allowed without permission.

554

\begin{tabular}{|c|c|c|c|c|}
\hline \multicolumn{5}{|c|}{ ROC-AUC } \\
\hline \multirow{2}{*}{ Goal of Comparison } & \multirow{2}{*}{ Model } & \multicolumn{3}{|c|}{ Benchmark } \\
\hline & & Dissimilar Protein & Kinase Inhibitor & Random \\
\hline DISAE & ALBERT frozen transformer & 0.725 & 0.690 & 0.889 \\
\hline the effect of pre-training & ALBERT frozen transformer (pre-trained on GPCR) & 0.441 & / & 0.849 \\
\hline the effect of distilled sequence & ALBERT frozen transformer (distilled singlets) & 0.583 & 1 & 0.656 \\
\hline \multirow{2}{*}{ the effect of fine-tuning } & ALBERT frozen embedding & 0.585 & / & 0.889 \\
\hline & ALBERT all unfrozen & 0.680 & T & 0.891 \\
\hline \multirow{3}{*}{ baseline against $A L B E R T$} & Transformer (distilled triplets) & 0.648 & 0.687 & 0.836 \\
\hline & LSTM (distilled singlets) & 0.652 & 0.642 & 0.907 \\
\hline & LSTM (distilled triplets) & 0.476 & 0.667 & 0.908 \\
\hline
\end{tabular}

\begin{tabular}{|c|c|c|c|c|}
\hline \multicolumn{5}{|c|}{ PR-AUC } \\
\hline \multirow{2}{*}{ Goal of Comparison } & \multirow{2}{*}{ Model } & \multicolumn{3}{|c|}{ Benchmark } \\
\hline & & Dissimilar Protein & Kinase Inhibitor & Random \\
\hline DISAE & ALBERT frozen transformer & 0.589 & 0.673 & 0.783 \\
\hline the effect of pre-training & ALBERT frozen transformer (pre-trained on GPCR) & 0.215 & / & 0.728 \\
\hline the effect of distilled sequence & ALBERT frozen transformer (distilled singlets) & 0.370 & / & 0.477 \\
\hline \multirow{2}{*}{ the effect of fine-tuning } & ALBERT frozen embedding & 0.278 & l & 0.783 \\
\hline & ALBERT all unfrozen & 0.418 & 1 & 0.785 \\
\hline \multirow{3}{*}{ baseline against ALBERT } & Transformer (distilled triplets) & 0.380 & 0.630 & 0.719 \\
\hline & LSTM (distilled singlets) & 0.372 & 0.614 & 0.798 \\
\hline & LSTM (distilled triplets) & 0.261 & 0.590 & 0.804 \\
\hline
\end{tabular}

Table 1: Test set performance under three benchmark settings evaluated in ROC-AUC and PR-AUC. ALBERT pre-trained transformer-frozen model outperforms other models, and its performance is stable across all settings. Hence, it is recommended as the optimal configuration for the pre-trained ALBERT model. Four variants of DISAE models are compared to the frozen transformer one. Unless specified in the parentheses, ALBERT is pre-trained on whole Pfam proteins in the form of distilled triplets. The four DISAE variants are organized into three groups based on the goal of comparison. Two state-of-the-art models Transformer and LSTM are compared with the ALBERT pre-trained models as baselines. Protein similarity based splitting uses a threshold of similarity score of 0.035 (Figure 2).

\begin{tabular}{|l|l|l|l|}
\hline Orphan Receptor (Uniprot Id) & Pfam & Drug & Drug Target \\
\hline A0A0C4DFX5 & PF13853 & Isoetharine & ADRB1, ADRB2 \\
\hline A0A126GVR8 & PF13853 & Ganirelix & GNRHR \\
\hline A0A1B0GTK7 & PF00001 & Levallorphan & OPRM1 \\
\hline A0A1B0GVZ0 & PF00001 & Xamoterol & ADRB1, ADRB2 \\
\hline A0A286YFH6 & PF13853 & Degarelix & GNRHR \\
\hline A3KFT3 & PF13853 & Degarelix & GNRHR \\
\hline C9J1J7 & PF00001 & Levallorphan & OPRM1 \\
\hline C9JQD8 & PF00001 & Lixisenatide & GLP1R \\
\hline E9PH76 & PF00001 & Ganirelix & GNRHR \\
\hline E9PPJ8 & PF13853 & Xamoterol & ADRB1, ADRB2 \\
\hline
\end{tabular}

Table 2: Example of deorphanization prediction from DISAE ensemble models. 
bioRxiv preprint doi: https://doi.org/10.1101/2020.10.08.332346; this version posted October 9, 2020. The copyright holder for this preprint (which was not certified by peer review) is the author/funder. All rights reserved. No reuse allowed without permission.

\section{Supplementary Information}

October 9, 2020 
bioRxiv preprint doi: https://doi.org/10.1101/2020.10.08.332346; this version posted October 9, 2020. The copyright holder for this preprint (which was not certified by peer review) is the author/funder. All rights reserved. No reuse allowed without permission.
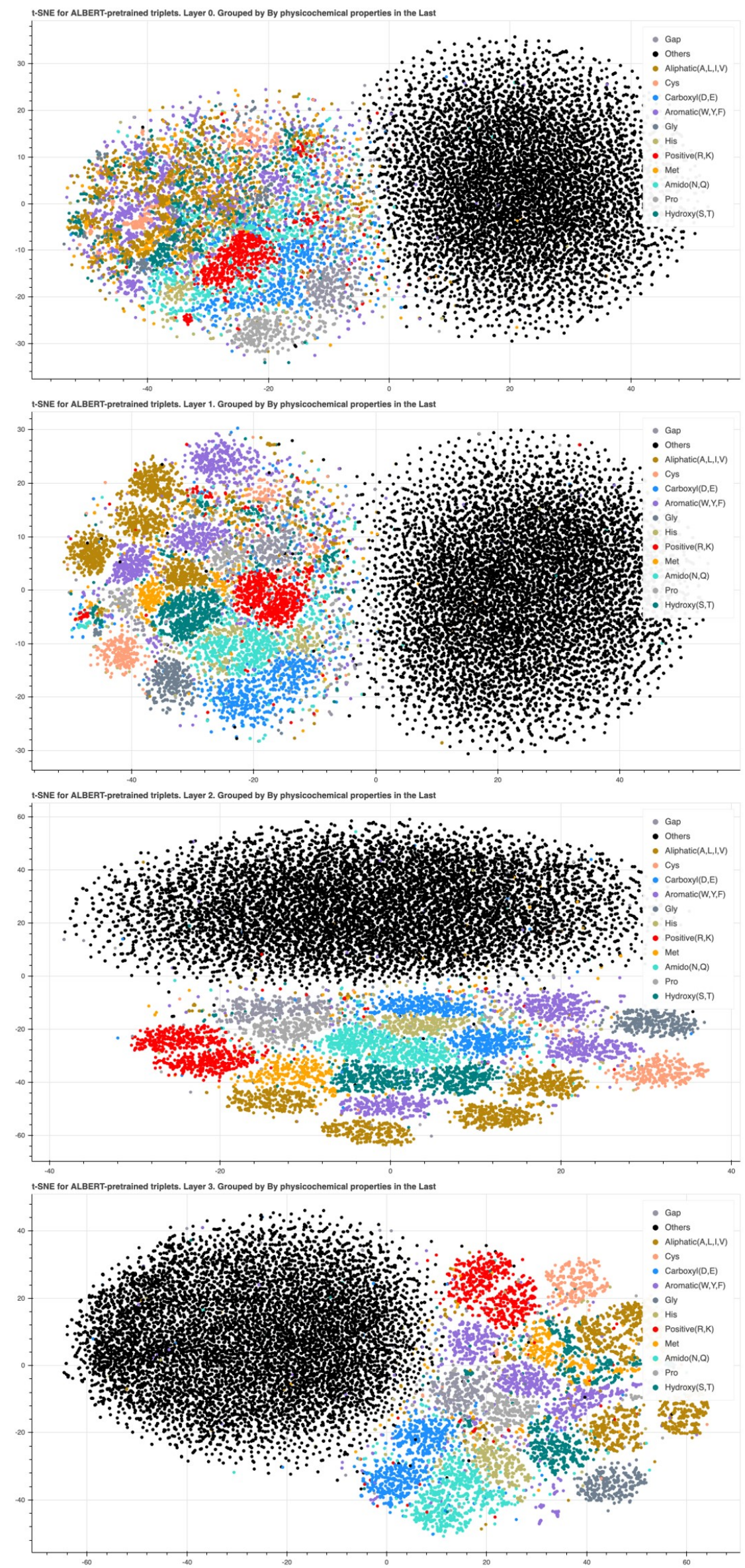

Supplementary Figure 1: Clustering of pre-trained triplet DISAE vectors at (A) Level 1, (B) Level 2, (C) Level 3, and (D) Level 4 of ALBERT. The triplet is colored by the physiochemical properties of the third amino acid. 
bioRxiv preprint doi: https://doi.org/10.1101/2020.10.08.332346; this version posted October 9, 2020. The copyright holder for this preprint (which was not certified by peer review) is the author/funder. All rights reserved. No reuse allowed without permission.
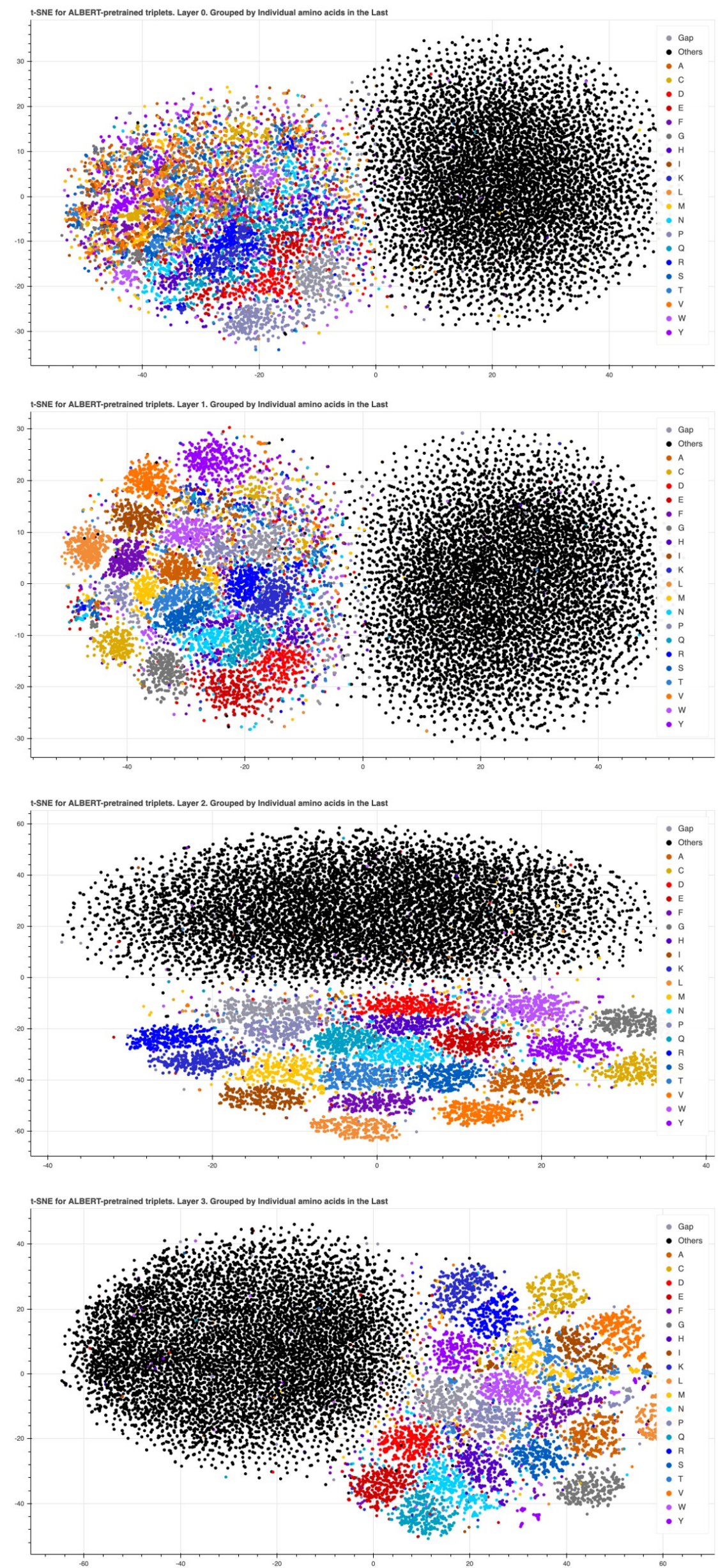

Supplementary Figure 2: The same clustering trends as in Supplementary Figure 1 were also observed when triplets are grouped by individual amino acid types, rather than their physicochemical properties of side chains. 3 

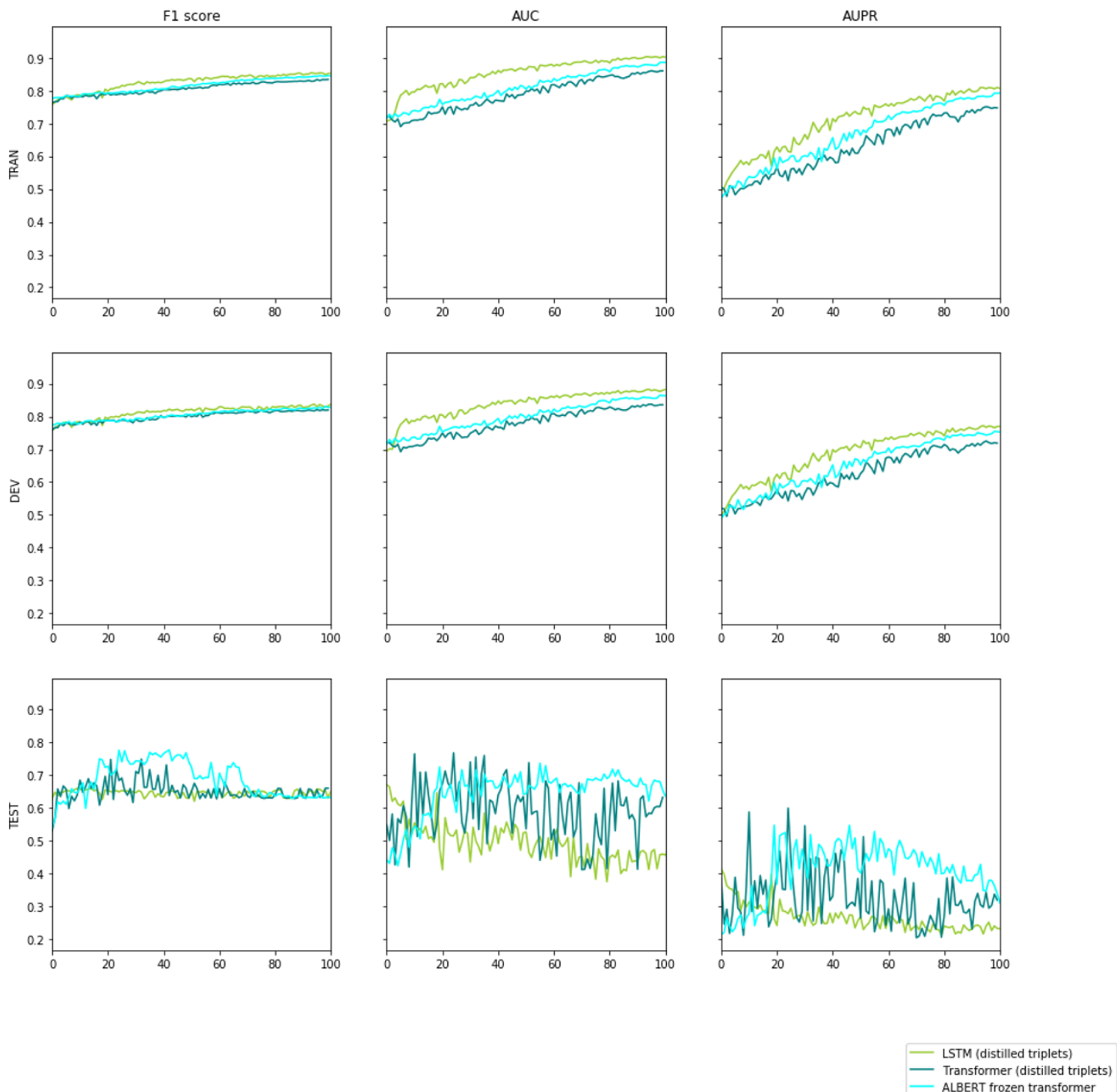

Supplementary Figure 3: The three major models as in the AUC curves in the paper. All $\mathrm{x}$ axis are the number of epochs trained. ALBERT frozen transformer proves consistent better performance, which becomes the critical advantage of DISAE in deorphanization, whhere remote orphan proteins are significantly different from training data and overfitting is impossible to control given the unknown true labels for classification. Using DISAE, we could be confident that even we train the model for too long or too short epochs, the prediction reliability on orphans will be robust. 

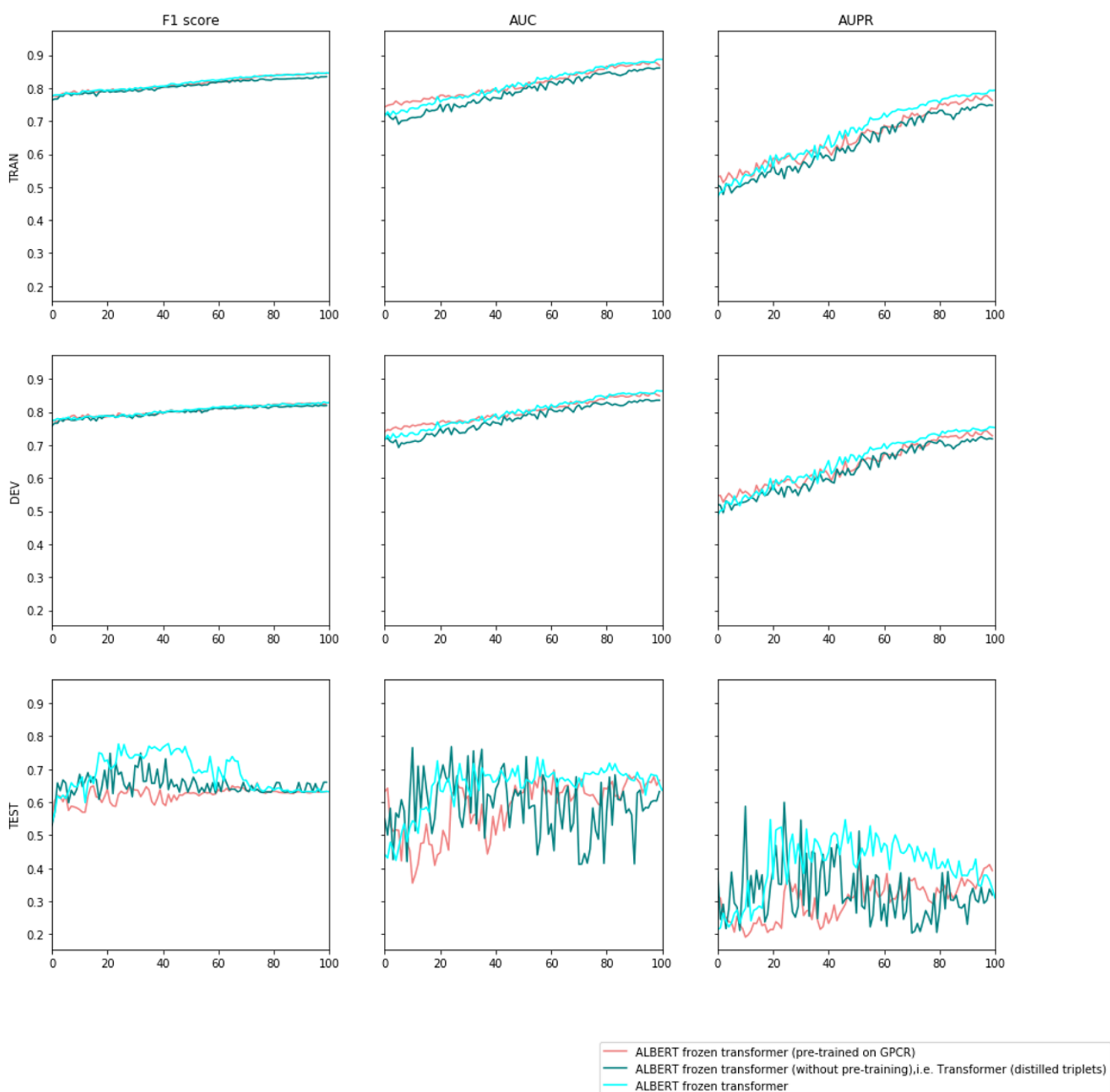

Supplementary Figure 4: The effect of pre-training: ALBERT frozen transformer, i.e. the one pre-trained on whole pfams as proposed in DISAE, show robust and consistent better performance. All $\mathrm{x}$ axis are the number of epochs trained. 

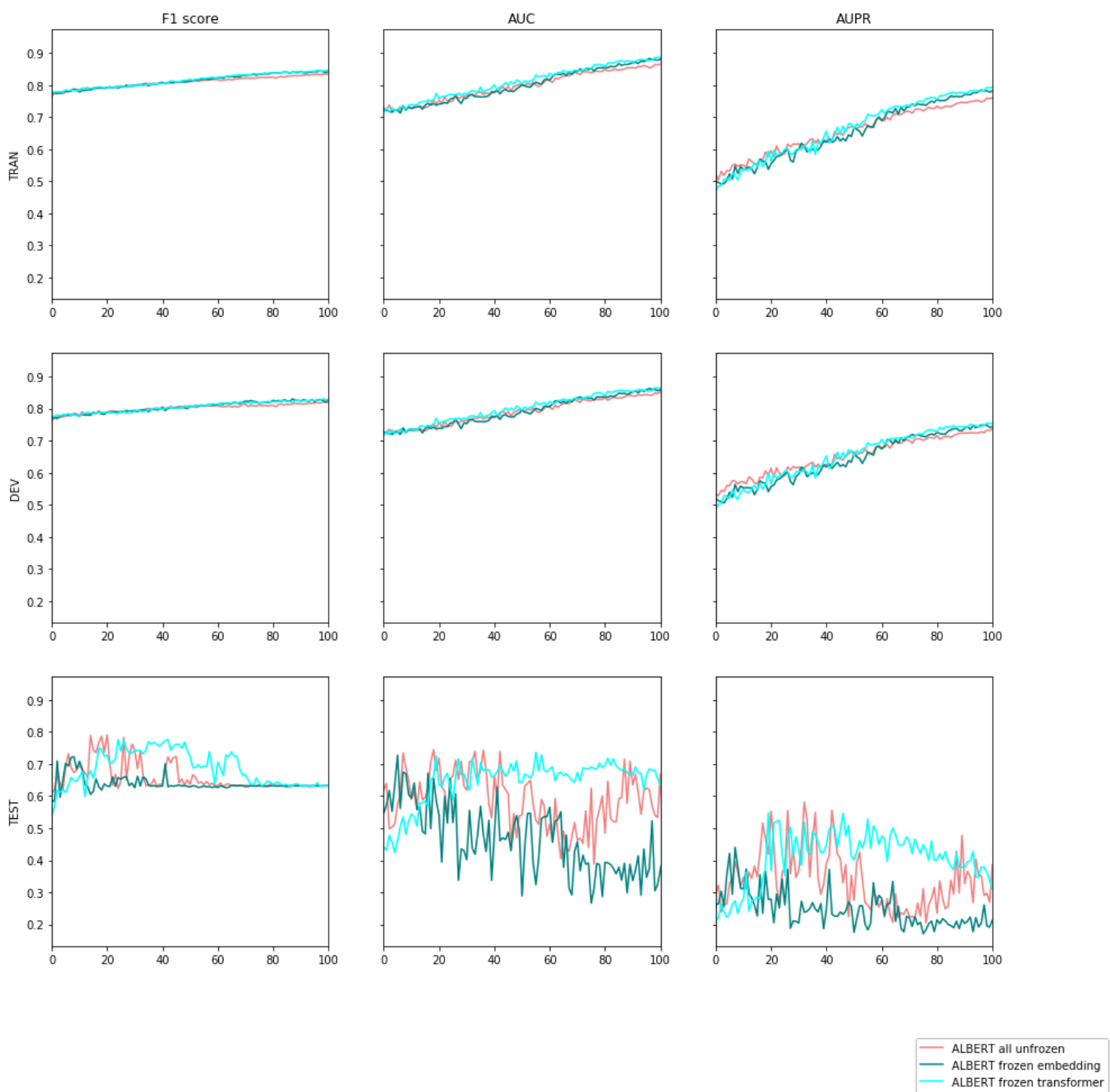

Supplementary Figure 5: The effect of different fine-tuning strategy with freezing parts of ALBERT: Although the other setting could be as good as ALBERT frozen transformer in some epochs, but they all suffer from large performance variance by epoch. ALBERT frozen transformer proves consistent and robust high performance. All $\mathrm{x}$ axis are the number of epochs trained 
bioRxiv preprint doi: https://doi.org/10.1101/2020.10.08.332346; this version posted October 9, 2020. The copyright holder for this preprint (which was not certified by peer review) is the author/funder. All rights reserved. No reuse allowed without permission.

PF03402

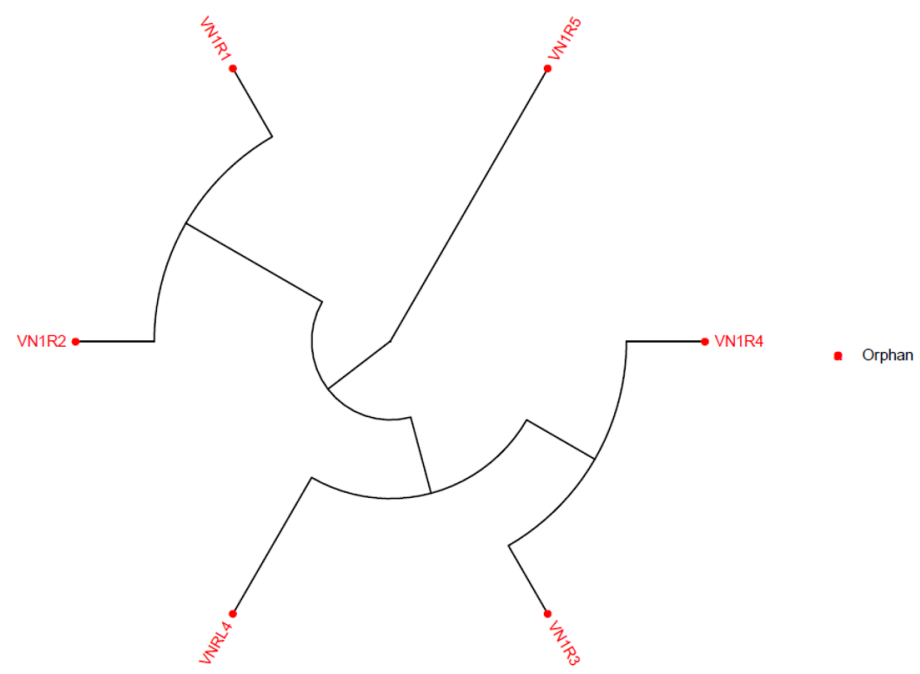

Supplementary Figure 6: Phylogenetic tree for PF03402 
bioRxiv preprint doi: https://doi.org/10.1101/2020.10.08.332346; this version posted October 9, 2020. The copyright holder for this preprint (which was not certified by peer review) is the author/funder. All rights reserved. No reuse allowed without permission.

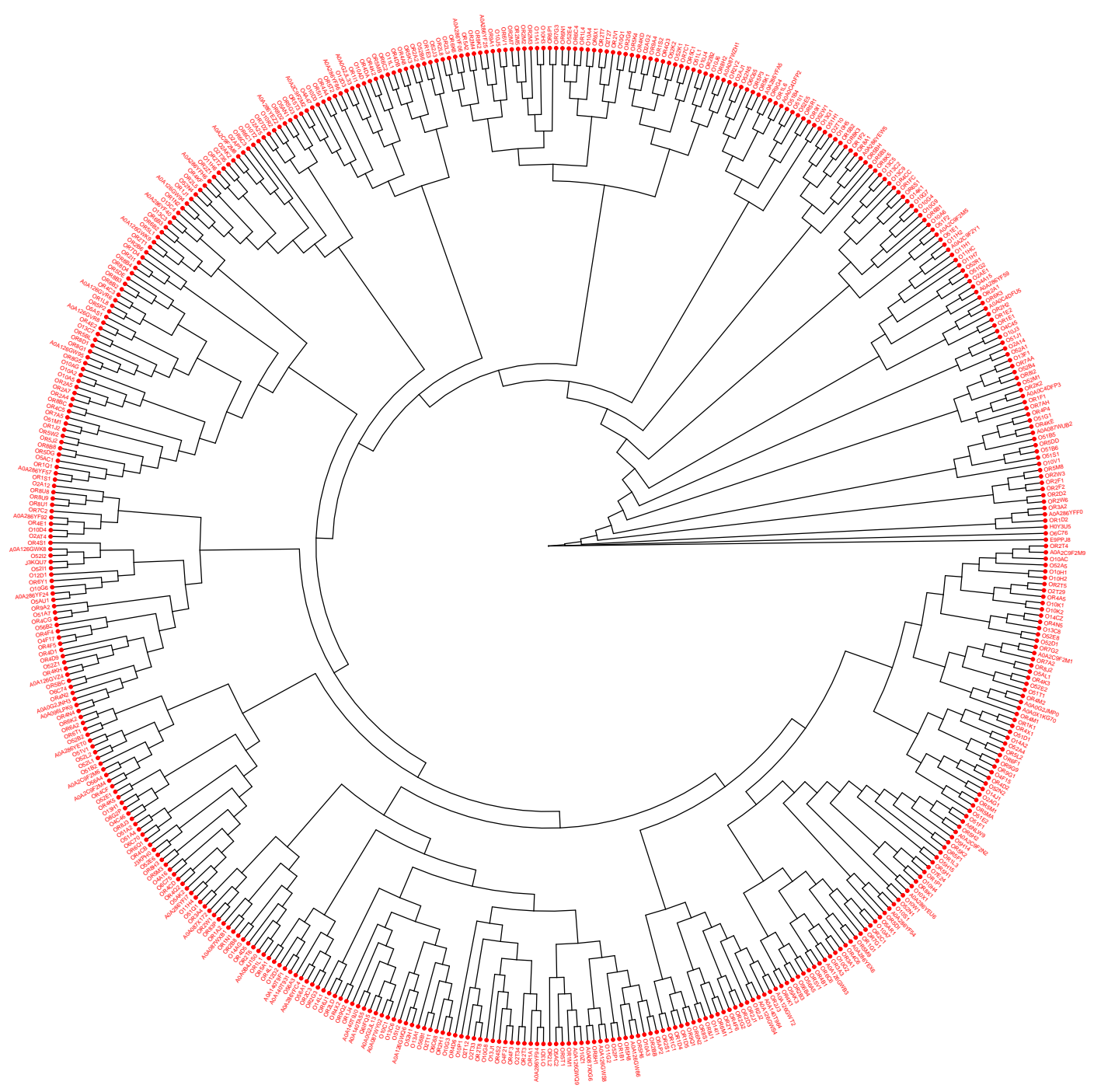

Supplementary Figure 7: Phylogenetic tree for PF13853 


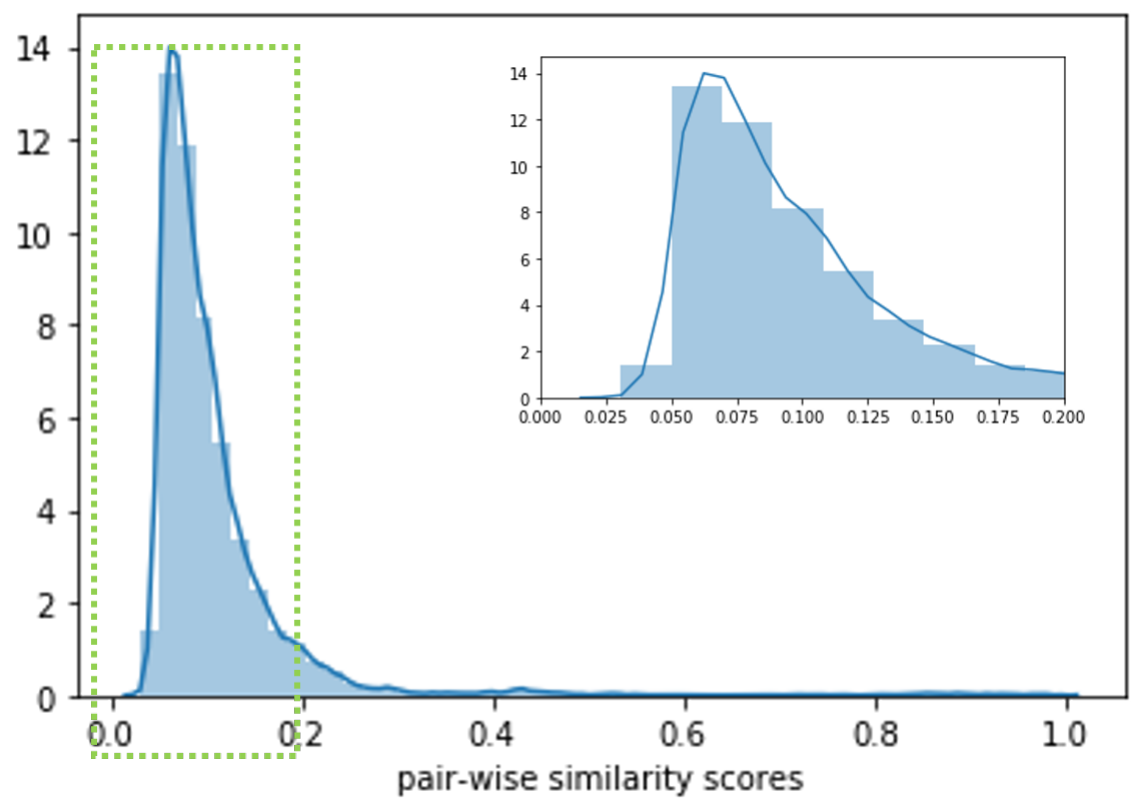

Supplementary Figure 8: Protein pair wise similarity distribution of benchmark data:To simulate realistic deorphanization scenario, where remote orphan protein could be significantly different from data used to train models, we design a protein similarity based data splitting strategy. First, pair wise bit-scores are calculated with standard BLAST+ package. A distribution could be found in Figure 8 Then, setting a threshold of 0.035, proteins with pair-wise similarity lower than the threshold are keep separately in two groups. The protein-chemical activity pairs with protein in the relative smaller group will be used as testing data. The left samples are then split into training and validation. 
bioRxiv preprint doi: https://doi.org/10.1101/2020.10.08.332346; this version posted October 9,2020 . The copyright holder for this preprint

(which was not certified by peer review) is the author/funder. All rights reserved. No reuse allowed without permission.

\begin{tabular}{|c|c|}
\hline Orphan Receptor & Predicted Drug [Known binding protein] \\
\hline A0A087WY02 & XXPANQJNYNUNES-UHFFFAOYSA-N ['P23975', 'Q05940', 'P31645'] \\
\hline A0A0A0MQW8 & BYJAVTDNIXVSPW-UHFFFAOYSA-N ['Q96RJ0', 'P35348', 'P43140' \\
\hline A0A0B4J1V8 & RGCVKNLCSQQDEP-UHFFFAOYSA-N ['P33261', 'P18901', 'P08684' \\
\hline A0A0C4DFX5 & MBUVEWMHONZEQD-UHFFFAOYSA-N ['P11509', 'P33261', 'Q16873'] \\
\hline A0A126GVR8 & BPZSYCZIITTYBL-YJYMSZOUSA-N ['P13945', 'P11509', 'P07550'] \\
\hline A0A126GWK9 & VQODGRNSFPNSQE-DVTGEIKXSA-N ['P46721', 'P04083', 'P20309' \\
\hline A0A126GWS4 & VMWNQDUVQKEIOC-CYBMUJFWSA-N ['P18901', 'P08684', 'P50226'] \\
\hline A0A1B0GTK7 & ATALOFNDEOCMKK-OITMNORJSA-N ['P29371', 'P11712', 'P33261'] \\
\hline A0A1B0GVZ0 & OWQUZNMMYNAXSL-UHFFFAOYSA-N ['Q01959', 'P31390', 'P35367'] \\
\hline A0A286YF86 & 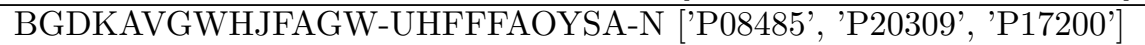 \\
\hline A0A286YF92 & IRSCQMHQWWYFCW-UHFFFAOYSA-N [", 'Q13255', 'Q96FL8'] \\
\hline A0A286YFH6 & BUGYDGFZZOZRHP-UHFFFAOYSA-N ['O00591', 'P11509', 'Q96FL8'] \\
\hline A0A2C9F2M5 & CYQFCXCEBYINGO-IAGOWNOFSA-N ['P47746', 'P47936', 'P11712'] \\
\hline A3KFT3 & KKZJGLLVHKMTCM-UHFFFAOYSA-N [", 'Q16678', 'P33527'] \\
\hline A6NFC9 & BYJAVTDNIXVSPW-UHFFFAOYSA-N ['Q96RJ0', 'P35348', 'P43140'] \\
\hline A6NH00 & BGDKAVGWHJFAGW-UHFFFAOYSA-N ['P08485', 'P20309', 'P17200'] \\
\hline A6NMS3 & IYIKLHRQXLHMJQ-UHFFFAOYSA-N ['P11509', 'P07550', 'P51589'] \\
\hline A6NMU1 & BARDROPHSZEBKC-OITMNORJSA-N ['P08684', 'P11712', 'P25103'] \\
\hline C9J1J7 & YFGHCGITMMYXAQ-LJQANCHMSA-N ['P20815', 'P11712', 'P05177'] \\
\hline C9JQD8 & LWAFSWPYPHEXKX-UHFFFAOYSA-N ['P10635', 'P07550', 'P08588'] \\
\hline C9JW47 & BYBLEWFAAKGYCD-UHFFFAOYSA-N ['Q6PIU1', 'P22001', 'P33261'] \\
\hline E7ENI1 & OCJYIGYOJCODJL-UHFFFAOYSA-N ['P10635', 'P02768', 'P35367'] \\
\hline E9PH76 & ZSCDBOWYZJWBIY-UHFFFAOYSA-N ['P07550', 'P33261', 'P11229'] \\
\hline E9PPJ8 & OZYUPQUCAUTOBP-QXAKKESOSA-N ['P33535', 'P35372'] \\
\hline F8VUV1 & CYQFCXCEBYINGO-IAGOWNOFSA-N ['P47746', 'P47936', 'P11712'] \\
\hline G3V4Q5 & XXPANQJNYNUNES-UHFFFAOYSA-N ['P23975', 'Q05940', 'P31645'] \\
\hline H0Y622 & 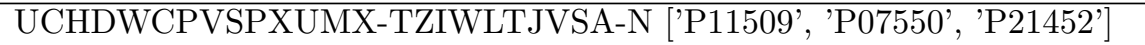 \\
\hline J3KQU7 & IZTQOLKUZKXIRV-YRVFCXMDSA-N ['Q9NPD5', 'P30553', 'Q63931'] \\
\hline O00590 & JOATXPAWOHTVSZ-UHFFFAOYSA-N ['P13945', 'P07550', 'P18089'] \\
\hline O15218 & BYJAVTDNIXVSPW-UHFFFAOYSA-N ['Q96RJ0', 'P35348', 'P43140' \\
\hline O43869 & UCHDWCPVSPXUMX-TZIWLTJVSA-N ['P11509', 'P07550', 'P21452' \\
\hline O60431 & XXPANQJNYNUNES-UHFFFAOYSA-N ['P23975', 'Q05940', 'P31645'] \\
\hline O76099 & SHGAZHPCJJPHSC-YCNIQYBTSA-N ['P31025', 'P11509', 'Q02928'] \\
\hline $\mathrm{P} 04000$ & LUZRJRNZXALNLM-JGRZULCMSA-N ['P08684', 'P34969', 'P35462'] \\
\hline P04001 & XXPANQJNYNUNES-UHFFFAOYSA-N ['P23975', 'Q05940', 'P31645'] \\
\hline P0C604 & DGBIGWXXNGSACT-UHFFFAOYSA-N ['O00591', 'O14764', 'P48169'] \\
\hline P0C628 & HTIQEAQVCYTUBX-UHFFFAOYSA-N ['Q02641', 'P08684', 'P04798'] \\
\hline P0C645 & IRSCQMHQWWYFCW-UHFFFAOYSA-N [", 'Q13255', 'Q96FL8'] \\
\hline P0C7N5 & 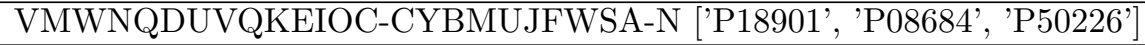 \\
\hline P0DMS8 & DGBIGWXXNGSACT-UHFFFAOYSA-N ['O00591', 'O14764', 'P48169'] \\
\hline P0DN77 & XXPANQJNYNUNES-UHFFFAOYSA-N ['P23975', 'Q05940', 'P31645'] \\
\hline P0DN78 & XXPANQJNYNUNES-UHFFFAOYSA-N ['P23975', 'Q05940', 'P31645'] \\
\hline $\mathrm{P} 47804$ & QWAXKHKRTORLEM-UGJKXSETSA-N ['P21452', 'P08684', 'P35462' \\
\hline $\mathrm{P} 58173$ & VHYCDWMUTMEGQY-UHFFFAOYSA-N ['P13945', 'P07550', 'P08588'] \\
\hline Q15612 & OIRDTQYFTABQOQ-KQYNXXCUSA-N ['P29275', 'P55263', 'P25099'] \\
\hline Q15617 & ZSCDBOWYZJWBIY-UHFFFAOYSA-N ['P07550', 'P33261', 'P11229'] \\
\hline Q6IFH4 & KWTSXDURSIMDCE-QMMMGPOBSA-N ['P25100', 'Q8HZ64', 'P10635' \\
\hline Q7Z5H5 & IYIKLHRQXLHMJQ-UHFFFAOYSA-N ['P11509', 'P07550', 'P51589' \\
\hline Q8N0Y5 & COUYJEVMBVSIHV-SFHVURJKSA-N ['P10632', 'P07550', 'P11712'] \\
\hline Q8N6U8 & BYJAVTDNIXVSPW-UHFFFAOYSA-N ['Q96RJ0', 'P35348', 'P43140'] \\
\hline Q8NG76 & IZTQOLKUZKXIRV-YRVFCXMDSA-N ['Q9NPD5', 'P30553', 'Q63931'] \\
\hline Q8NG83 & CJOFXWAVKWHTFT-XSFVSMFZSA-N ['Q9HB55', 'P22086', 'P10635'] \\
\hline Q8NG85 & IYIKLHRQXLHMJQ-UHFFFAOYSA-N ['P11509', 'P07550', 'P51589'] \\
\hline Q8NGB2 & BARDROPHSZEBKC-OITMNORJSA-N ['P08684', 'P11712', 'P25103'] \\
\hline Q8NGC2 & MEFKEPWMEQBLKI-AIRLBKTGSA-N ['P19623', 'P31153', 'P25099' \\
\hline Q8NGC8 & COUYJEVMBVSIHV-SFHVURJKSA-N ['P10632', 'P07550', 'P11712'] \\
\hline Q8NGD3 & OCJYIGYOJCODJL-UHFFFAOYSA-N ['P10635' \\
\hline
\end{tabular}


Table 1 continued from previous page

\begin{tabular}{|c|c|}
\hline Orphan Receptor & Predicted Drug [Known binding protein] \\
\hline Q8NGD4 & UCTWMZQNUQWSLP-VIFPVBQESA-N ['P07550', 'O15244', 'P08684' \\
\hline Q8NGE9 & WNTYBHLDCKXEOT-UHFFFAOYSA-N ['P14416', 'P31388', 'P10275' \\
\hline Q8NGG2 & BGDKAVGWHJFAGW-UHFFFAOYSA-N ['P08485', 'P20309', 'P17200' \\
\hline Q8NGI3 & CYQFCXCEBYINGO-IAGOWNOFSA-N ['P47746', 'P47936', 'P11712'] \\
\hline Q8NGI4 & IRSCQMHQWWYFCW-UHFFFAOYSA-N [", 'Q13255', 'Q96FL8'] \\
\hline Q8NGI7 & OCJYIGYOJCODJL-UHFFFAOYSA-N ['P10635', 'P02768', 'P35367'] \\
\hline Q8NGJ5 & JSWZEAMFRNKZNL-UHFFFAOYSA-N ['P11712', 'P41595', 'P05177' \\
\hline Q8NGJ9 & GEFQWZLICWMTKF-CDUCUWFYSA-N ['P25100', 'P18089', 'P08913'] \\
\hline Q8NGK2 & LUZRJRNZXALNLM-JGRZULCMSA-N ['P08684', 'P34969', 'P35462'] \\
\hline Q8NGK6 & ZSCDBOWYZJWBIY-UHFFFAOYSA-N ['P07550', 'P33261', 'P11229' \\
\hline Q8NGL9 & UCHDWCPVSPXUMX-TZIWLTJVSA-N ['P11509', 'P07550', 'P21452' \\
\hline Q8NGM9 & BYJAVTDNIXVSPW-UHFFFAOYSA-N ['Q96RJ0', 'P35348', 'P43140' \\
\hline Q8NGN7 & XXPANQJNYNUNES-UHFFFAOYSA-N ['P23975', 'Q05940', 'P31645' \\
\hline Q8NGR4 & BGDKAVGWHJFAGW-UHFFFAOYSA-N ['P08485', 'P20309', 'P17200'] \\
\hline Q8NGS1 & XXPANQJNYNUNES-UHFFFAOYSA-N ['P23975', 'Q05940', 'P31645'] \\
\hline Q8NGT5 & ATALOFNDEOCMKK-OITMNORJSA-N ['P29371', 'P11712', 'P33261' \\
\hline Q8NGU2 & UCHDWCPVSPXUMX-TZIWLTJVSA-N ['P11509', 'P07550', 'P21452'] \\
\hline Q8NGW1 & DRHKJLXJIQTDTD-OAHLLOKOSA-N ['P08684', 'P34969', 'P35462'] \\
\hline Q8NGX9 & URKOMYMAXPYINW-UHFFFAOYSA-N ['P31389', 'P07550', 'P33261' \\
\hline Q8NGY9 & UCTWMZQNUQWSLP-VIFPVBQESA-N ['P07550', 'O15244', 'P08684' \\
\hline Q8NGZ2 & IZTQOLKUZKXIRV-YRVFCXMDSA-N ['Q9NPD5', 'P30553', 'Q63931'] \\
\hline Q8NGZ4 & RUDATBOHQWOJDD-BSWAIDMHSA-N ['P52895', 'Q96RI1', 'P08684'] \\
\hline Q8NH04 & BYBLEWFAAKGYCD-UHFFFAOYSA-N ['Q6PIU1', 'P22001', 'P33261'] \\
\hline Q8NH05 & KKGQTZUTZRNORY-UHFFFAOYSA-N ['P21453', 'O95977', 'P43004' \\
\hline Q8NH41 & OZVBMTJYIDMWIL-AYFBDAFISA-N ['P07550', 'P08684', 'P34969' \\
\hline Q8NH48 & ZSCDBOWYZJWBIY-UHFFFAOYSA-N ['P07550', 'P33261', 'P11229' \\
\hline Q8NH51 & BUGYDGFZZOZRHP-UHFFFAOYSA-N ['O00591', 'P11509', 'Q96FL8'] \\
\hline Q8NH57 & XXPANQJNYNUNES-UHFFFAOYSA-N ['P23975', 'Q05940', 'P31645'] \\
\hline Q8NH61 & RUDATBOHQWOJDD-BSWAIDMHSA-N ['P52895', 'Q96RI1', 'P08684'] \\
\hline Q8NH74 & BYJAVTDNIXVSPW-UHFFFAOYSA-N ['Q96RJ0', 'P35348', 'P43140'] \\
\hline Q8NH87 & GJPICJJJRGTNOD-UHFFFAOYSA-N ['P25101', 'P11712', 'O95342'] \\
\hline Q8NH89 & ZSCDBOWYZJWBIY-UHFFFAOYSA-N ['P07550', 'P33261', 'P11229' \\
\hline Q8NH95 & DERZBLKQOCDDDZ-JLHYYAGUSA-N ['P11509', 'O60840', 'P11229' \\
\hline Q8TCB6 & HTIQEAQVCYTUBX-UHFFFAOYSA-N ['Q02641', 'P08684', 'P04798' \\
\hline Q8TDV2 & XXPANQJNYNUNES-UHFFFAOYSA-N ['P23975', 'Q05940', 'P31645' \\
\hline Q8WZA6 & ZFXYFBGIUFBOJW-UHFFFAOYSA-N ['P29275', 'P25099', 'Q60614' \\
\hline Q96KK4 & XXPANQJNYNUNES-UHFFFAOYSA-N ['P23975', 'Q05940', 'P31645' \\
\hline Q96R54 & VMWNQDUVQKEIOC-CYBMUJFWSA-N ['P18901', 'P08684', 'P50226'] \\
\hline Q96R67 & ZSCDBOWYZJWBIY-UHFFFAOYSA-N ['P07550', 'P33261', 'P11229'] \\
\hline Q9BZJ7 & UCHDWCPVSPXUMX-TZIWLTJVSA-N ['P11509', 'P07550', 'P21452' \\
\hline Q9BZJ8 & KWTSXDURSIMDCE-QMMMGPOBSA-N ['P25100', 'Q8HZ64', 'P10635' \\
\hline Q9GZM6 & $\begin{array}{l}\text { PVNIIMVLHYAWGP-UHFFFAOYSA-N ['P49019', 'Q15274', 'Q80Z39'] } \\
\end{array}$ \\
\hline Q9H1Y3 & FIVSJYGQAIEMOC-ZGNKEGEESA-N ['P10635', 'Q9UNQ0', 'P25103'] \\
\hline Q9H210 & IYIKLHRQXLHMJQ-UHFFFAOYSA-N ['P11509', 'P07550', 'P51589' \\
\hline Q9H339 & IYIKLHRQXLHMJQ-UHFFFAOYSA-N ['P11509', 'P07550', 'P51589' \\
\hline Q9NZP2 & IQVRBWUUXZMOPW-PKNBQFBNSA-N ['P29275', 'Q96FL8', 'P25099'] \\
\hline Q9UGF5 & GHOSNRCGJFBJIB-UHFFFAOYSA-N ['P30556', 'P11712', 'P23219'] \\
\hline Q9UGF6 & BTCSSZJGUNDROE-UHFFFAOYSA-N ['Q7Z2H8', 'Q9Z0U4', 'Q9UBS5'] \\
\hline Q9UHM6 & BGDKAVGWHJFAGW-UHFFFAOYSA-N ['P08485', 'P20309', 'P17200' \\
\hline
\end{tabular}

Supplementary Table 1: Predicted approved drug examples: 649 human orphan GPCRs, each paired to 555 approved GPCR-targeted drugs as novel samples chemical-protein pairs. Listed here are 106 of the orphan proteins paired with at least one approved GPCRtargeted drugs with estimated false positive rate lower than 0.05. Proteins are presented with Uniprot Id and chemicals are presented with InChIKey. 


\begin{tabular}{|c|c|c|c|}
\hline & & \multicolumn{2}{|c|}{ ALBERT MODEL CONFIGURATION } \\
\hline & & TRIPLETS FORM & SINGLET FORM \\
\hline \multirow{19}{*}{ pre-training related } & "attention_probs_dropout_prob" & \multicolumn{2}{|c|}{0} \\
\hline & "hidden_act": "gelu" & \multicolumn{2}{|c|}{ "gelu" } \\
\hline & "hidden_dropout_prob" & \multicolumn{2}{|c|}{0} \\
\hline & "embedding_size" & \multicolumn{2}{|c|}{128} \\
\hline & "hidden_size" & \multicolumn{2}{|c|}{312} \\
\hline & "initializer_range" & \multicolumn{2}{|c|}{0.02} \\
\hline & "intermediate_size" & \multicolumn{2}{|c|}{1248} \\
\hline & "max_position_embeddings" & \multicolumn{2}{|c|}{512} \\
\hline & "num_attention_heads" & \multicolumn{2}{|c|}{12} \\
\hline & "num_hidden_layers" & \multicolumn{2}{|c|}{4} \\
\hline & "num_hidden_groups" & \multicolumn{2}{|c|}{1} \\
\hline & "net_structure_type" & \multicolumn{2}{|c|}{0} \\
\hline & "gap_size" & \multicolumn{2}{|c|}{0} \\
\hline & "num_memory_blocks" & \multicolumn{2}{|c|}{0} \\
\hline & "inner_group_num" & \multicolumn{2}{|c|}{1} \\
\hline & "down_scale_factor" & \multicolumn{2}{|c|}{1} \\
\hline & "type_vocab_size" & \multicolumn{2}{|c|}{2} \\
\hline & "vocab_size" & 19686 & 32 \\
\hline & "ln_type" & 1 & "postIn" \\
\hline \multirow{2}{*}{ fine-tuning related } & sequence embedding hidden units & \multicolumn{2}{|c|}{256} \\
\hline & protein sequence post-tokenization length & \multicolumn{2}{|c|}{210} \\
\hline
\end{tabular}

Supplementary Table 2: ALBERT configuration. ALBERT is using the package Transformers by Huggingface (https://github.com/huggingface/transformers). The author installed the package in Jan 2020.

\begin{tabular}{|l|l|}
\hline \multicolumn{2}{|c|}{ Neural-fingerprint CONFIGURATION } \\
\hline Dropout & 0.1 \\
\hline converlution layer size & 20 \\
\hline converlution layer number & 4 \\
\hline hidden units & 128 \\
\hline Atomic connectivity degrees for chemical molecules & {$[0,1,2,3,4,5]$} \\
\hline \multicolumn{1}{|c|}{ INERACTION PREDICTION CONFIGURATION } \\
\hline design & two linear layer with batch normlization and ReLU \\
\hline attentive pooling drop out & 0.3 \\
\hline attentive pooling hidden units & 64 \\
\hline \multicolumn{2}{|c|}{ LSTM CONFIGURATION } \\
\hline embedding hidden units & 128 \\
\hline number of LSTM layers & 1 \\
\hline dropout & 0.2 \\
\hline
\end{tabular}

Supplementary Table 3: There are three major components of the fine tuning model architecture: ALBERT to extract protein embedding, Neural-fingerprint to extract chemical embedding, and interaction prediction layers. LSTM serves as the baseline.

\begin{tabular}{|l|l|}
\hline \multicolumn{2}{|l|}{ OPTIMIZATION CONFIGURATION } \\
\hline training epochs & 100 \\
\hline batch size & 64 \\
\hline optimizer & Adam \\
\hline scheduler to adjust learning rate & cosineannealing \\
\hline initial learnin rate & $2.00 \mathrm{E}-05$ \\
\hline L2 regularization weight & $1.00 \mathrm{E}-04$ \\
\hline Deep Learning Server & Supermicro SuperServer 4028GR-TR \\
\hline GPU & NVIDIA Teslaß V100 with 32 GB per GPU (256 GB total) of GPU memory \\
\hline CPU & Intel Xeon E5-2650 v4 2.2 GHz 12-Core (48-core total) \\
\hline
\end{tabular}

Supplementary Table 4: Optimization related configuration 